\title{
Psychological interventions: Overview and critical issues for the field
}

\section{Citation}

Weisz, John R., Mei Yi Ng, and Nancy Lau. 2015. Psychological interventions: Overview and critical issues for the field. In Rutter's Child and Adolescent Psychiatry, 6th Ed., eds. A. Tharpar, D. Pine, J. Leckman, S. Scott, M. Snowling, and E. Taylor. Chichester West Sussex, UK: John Wiley \& Sons.

\section{Published Version}

http://www.wiley.com/WileyCDA/WileyTitle/productCd-1118381963.html

\section{Permanent link}

http://nrs.harvard.edu/urn-3:HUL.InstRepos:34331452

\section{Terms of Use}

This article was downloaded from Harvard University's DASH repository, and is made available under the terms and conditions applicable to Open Access Policy Articles, as set forth at http:// nrs.harvard.edu/urn-3:HUL.InstRepos:dash.current.terms-of-use\#OAP

\section{Share Your Story}

The Harvard community has made this article openly available.

Please share how this access benefits you. Submit a story.

\section{Accessibility}


Chapter draft for Rutter's Child and Adolescent Psychiatry, $6^{\text {th }}$ Edition

Chapter 37. Psychological Interventions: Overview and Critical Issues for the Field

\author{
John R. Weisz \\ Professor, Psychology Department, Harvard University \\ Professor, Harvard Medical School \\ Mei Yi Ng \\ Graduate Student, Harvard University \\ Nancy Lau \\ Graduate Student, Harvard University
}

First Draft: Submitted April 30, 2013

Second Draft: Submitted October 15, 2013

Total word count in text and references: 14,775

Contact author: John R. Weisz, Department of Psychology, Harvard University, William James Hall, 33 Kirkland Street, Cambridge, MA 02138 


\title{
Psychological Interventions: Overview and Critical Issues for the Field
}

\author{
Abstract (229 words) \\ Efforts to help young people have ancient historical roots, but "youth psychotherapy" has only \\ been practiced for about a century and studied empirically for 50 years. Nonetheless, hundreds of \\ randomized trials have now accumulated, and many specific treatments have sufficient empirical \\ support that they are classified as evidence-based psychotherapies (EBPs). We examine the \\ strength of that support, highlight specific EBPs for specific forms of youth dysfunction, and \\ consider strategies for understanding how, with which groups, and under what conditions, the \\ treatments produce beneficial effects. We also examine how specific these effects are (e.g., \\ whether benefit is greatest for targeted symptoms), how robust (e.g., whether "blind" informants \\ perceive benefit, whether effects vary with type of control group), and how effective when \\ extended to youths in ethnic minority groups, low- and middle-income countries, war-torn \\ regions, and the criminal justice and child protection systems. We discuss the challenge of \\ disseminating EBPs and implementing them within the everyday practice of youth mental health \\ care. Strategies for improving youth psychotherapies and intervention science are proposed, \\ including making intervention research look more like clinical practice, leveraging the heuristic \\ potential of usual clinical care, conducting more high-risk/high-gain research and finding ways \\ to learn from treatment "failure," restructuring EBPs to fit clinical practice, boosting treatment \\ effectiveness through monitoring and feedback, enriching our understanding of what makes \\ treatments work, and building efficient and accessible delivery methods and models.
}

Key Words: youth, psychotherapy, evidence-based, mental health, meta-analysis, mechanisms of change, mediators, moderators, dissemination, implementation 


\section{Psychological Interventions: Overview and Critical Issues for the Field}

Psychological intervention has deep historical roots. These extend at least to ancient religious teachings about values for proper living, to Greek philosophers' use of discourse to alter perspectives and sharpen understanding, and to the long evolution of medical science and the healing traditions. Interventions for children and adolescents (henceforth "youth") share these ancient roots, but designation of youth psychotherapy as a distinctive specialty owes much to Sigmund Freud (1856-1939), notably his analysis of "Little Hans's" horse phobia and his psychoanalysis of his own daughter, Anna (1895-1982). Subsequent growth of youth psychotherapy was fueled by Freud's intellectual heirs, by the grand theories of personality and development, and by models and methods as different from psychoanalysis as behaviorism and cognitive psychology. By the end of the $20^{\text {th }}$ century, Kazdin (2000) had identified 551 different named therapies used with children and adolescents. Of course many more therapies are used in clinical practice, as hundreds of thousands of unique practitioners shape their own approaches.

While youth psychotherapy practice accelerated fast over the past century, psychotherapy research developed slowly. In 1952, Eysenck's influential review of adult psychotherapy studies suggested that therapy might be ineffective. Levitt's $(1957,1963)$ reviews, which included youth treatment, reached a similar conclusion. Since the time of Eysenk and Levitt, youth treatment outcome research has grown more rigorous, and its volume has mushroomed (see Silverman \& Hinshaw 2008; Weisz \& Kazdin 2010).

\section{Studying the Effects of Youth Psychotherapy: Methods and Findings}

Youth treatment effects are often assessed via randomized controlled trials (RCTs), and these RCTs can be pooled and synthesized within meta-analyses (discussed below). Multiple baseline designs, ABAB (sometimes called "reversal") designs, and other single-subject methods are also useful. These methods (see Barlow et al. 2009; Kazdin 2011) have been used in diverse 
treatment situations - including programs for ADHD (see, e.g., Pelham et al. 2010), studies of intervention for an entire classroom (see e.g., Wurtele \& Drabman 1984), or for only one or two youngsters with a rare condition (e.g., McGrath et al. 1987; Tarnowski et al.1987). Studies using these designs have produced a rich body of outcome evidence and some meta-analyses - e.g., on treatment for disruptive behavior (Chen \& Ma 2007), autism spectrum disorders (Campbell 2003), and social skill deficits (Mathur et al.1998). That said, youth psychotherapy metaanalyses have mainly focused on RCTs.

\section{Meta-analytic Findings}

Among the meta-analyses of youth RCTs, four have synthesized findings from diverse treatments and treated conditions. Casey and Berman (1985) focused on studies with children aged 12 and younger. Weisz et al. (1987) included studies with 4-18 year-olds. Kazdin et al. (1990) included studies with 4-18 year-olds. Finally, Weisz et al. (1995) spanned ages 2-18. Mean effect sizes found in these four meta-analyses are shown in Figure 1, with a comparison to two widely cited meta-analyses of predominantly adult psychotherapy (Smith \& Glass 1977; Shapiro \& Shapiro 1982). The figure shows that youth treatment effect sizes fall mainly within the range found for adult therapy, and on average between Cohen's (1988) benchmarks for medium (i.e., .5) and large (.8) effects.

Two other youth meta-analytic findings sharpen the picture in an encouraging way. First, findings (in Weisz et al.1987 and 1995) showed that effects measured immediately after treatment were quite similar to effects measured at follow-up assessments, which averaged about 6 months after the end of treatment. This suggests reasonable holding power of treatment benefit over time. Second, findings indicated that effects are larger for the specific problems targeted in treatment than for problems that were not specifically addressed (Weisz et al.1995, p. 460). This 
suggests that the tested therapies didn't just create nonspecific good feelings but instead impacted rather precisely the kinds of dysfunction they were designed to treat.

These findings suggest certain strengths of youth psychotherapies, but meta-analysis can also highlight challenges, critical questions, and areas in which intervention needs to be strengthened. A meta-analysis of psychotherapy for youth depression, for example, showed beneficial effects that were statistically significant (mean effect size: .34) but also significantly smaller than effects of treatments for other youth problems and disorders; even the very popular cognitive approaches, including CBT, were no more successful than alternate treatments in alleviating depression (see Weisz et al. 2006b). Such findings provide a reality check, potentially suggesting a need to strengthen or even re-envision youth depression treatment.

\section{Identifying "Evidence-Based Psychotherapies"}

As treatment outcome evidence accumulated over the years, and documentation of the tested treatments grew more precise (typically in the form of detailed treatment manuals), efforts were launched to identify the specific therapies sufficiently well-supported to be considered “evidence-based." Task forces and review teams were formed (see e.g., Chambless, et al.1998) to distill the research evidence and identify therapies that met standards for different levels of empirical support. Building on this work, experts in youth psychotherapy compiled their own reports in two journal special issues (Lonigan et al.1998; Silverman \& Hinshaw 2008). In the most recent report (Silverman \& Hinshaw 2008), reviewers identified therapies at four levels of empirical support: (1) well-established (e.g., two good group-design experiments by different research teams in two different settings, showing the treatment to be "superior to pill, psychological placebo, or another treatment”), (2) probably efficacious (e.g., “....at least two good experiments showing the treatment is superior...to a wait-list control group"), (3) possibly 
efficacious (e.g., "At least one 'good' study showing the treatment to be efficacious in the absence of conflicting evidence"), or (4) experimental (“...not yet tested in trials meeting task force criteria").

This 2008 report identified dozens of youth psychotherapies as either well-established or probably efficacious, encompassing multiple disorders and problems. Table 1 shows the youth treatments classified at these two highest levels in the Silverman-Hinshaw (2008) special issue.

\section{The Evidence-Based Treatments: "Well-Established" and "Probably Efficacious"}

Table 1 lists those interventions rated probably efficacious or well-established. Throughout this chapter, we will refer to these interventions as evidence-based psychotherapies (EBPs).

Autism. In the autism review (Rogers \& Vismara 2008), only one treatment was designated well-established: Ivar Lovaas's Early Intensive Behavioral Intervention (EIBI; Smith 2010); this is a form of applied behavior analysis that entails individual discreet trials training, often 30 hours per week or more, building specific core skills in language, social interaction, self-help, and other domains critical to children's development. Therapists treat the children and train parents to continue the intervention at home. A related intervention, Pivotal Response Training (PRT; Koegel et al.2010) was rated probably efficacious; PRT uses core skills of the Lovaas model but with an expanded emphasis on treatment in the child's natural environment and on strategies for boosting the child's motivation to learn (e.g., relying on activities the child chooses, identifying natural reinforcers). PRT teaches communication, academic, self-help, social, and even recreational skills; like EIBI, PRT relies heavily on training parents to deliver the intervention.

Eating disorders. Only one treatment for eating disorders was identified as an EBP (see Keel \& Haedt 2008): family therapy for anorexia nervosa, and in particular the Maudsley model (Lock 
et al. 2001). This model includes (a) relying on family members to return the client to more normal eating behavior - a process called "refeeding the client;" (b) negotiating restructured relationships in areas that influence eating behavior [e.g., if deceit is used by the family to avoid conflict and by the youth to conceal bingeing, therapy may focus on alternatives to deceit]; and (c) termination, including planning ways to sustain healthy eating by sustaining healthy intrafamily relationships and youth autonomy. No EBP was identified for bulimia nervosa in adolescents, but the reviewers (Keel \& Haedt 2008) noted that CBT (e.g., addressing distorted cognitions about body shape and size, using behavioral procedures to structure healthy eating habits) is a well-established treatment for bulimia in young adults and older adolescents. A special challenge in the eating domain is that eating disorders can take multiple forms, and in fact the most prevalent diagnostic category is "not otherwise specified." Thus, even effective treatments for anorexia and bulimia will not necessarily fit most eating disordered youths.

Anxiety disorders. For phobic and anxiety disorders in youth (reviewed by Silverman et al. 2008b), the most thoroughly tested psychotherapies are the CBTs; these most often blend graduated exposure to feared stimuli with efforts to identify and modify distorted cognitions that can spark and sustain fears. The treatments rated as EBPs included individual CBT, group CBT, group CBT with parents, and Social Effectiveness Therapy (SET) for Children (Beidel et al. 2000). SET, designed for social phobia, includes group sessions for youngsters, in-vivo exposure sessions (e.g., practicing interacting with non-anxious peers), and teaching parents to notice and reward their children's steps toward less anxious behavior in social situations.

In their review of interventions for obsessive-compulsive disorder, Barrett et al. 2008 identified only one treatment that met EBP criteria: individual exposure-based CBT. Youths are exposed to stimuli that lead to their obsessive fears but then told not to engage in the compulsive 
behavior those fears usually trigger. Ideally, across repeated exposures, the anxiety-obsessioncompulsion cycle is weakened via autonomic habituation and altered cognition, as youths learn that bad things they feared do not actually happen, even when they say no to their compulsions.

Finally, reviewing treatments for youths exposed to traumatic events, Silverman et al. (2008a) identified Trauma-Focused CBT (Cohen et al. 2010) as an EBP. This treatment, designed for young victims of sexual abuse and other forms of maltreatment, uses components of CBT for anxiety, plus additional elements designed to fit the situations in which trauma occurred. The components include, for example, safety planning (to reduce environmental risk) and "trauma narrative," in which young people write, then read, descriptions of their traumatic experiences. The draft narrative is repeatedly read to the therapist, as a form of exposure therapy. Distorted cognitions in the narrative (e.g., inappropriate self-blame) are modified through discussion by the youth and therapist. Caregivers participate, by joining the youth and therapist at later readings of the narrative and by supporting the youth's courage in sharing the story. Silverman et al. (2008a) also identified school-based group CBT (Kataoka et al. 2003; Stein et al. 2003) as an EBP. This treatment, designed for youths exposed to community violence, includes psychoeducation, cognitive work, coping skills training, social skills training, and graduated exposures carried out through writing and/or drawing.

Depression. David-Ferdon and Kaslow (2008), reviewing depression treatments, identified two broad approaches as EBPs: Interpersonal Psychotherapy for Adolescents (IPT-A; Mufson et al. 1999) and CBT. IPT-A focuses on interpersonal issues that are common among adolescents, such as the ways roles shift in the parent-teen relationship as teens mature; the intervention focuses on helping adolescents build effective strategies for dealing with role transitions, disputes, grief, and interpersonal deficits. As for CBT, its content varies across programs, but it 
typically includes such components as identifying and scheduling mood-boosting activities, identifying and modifying unrealistic negative thoughts, self-calming and relaxation, and building problem-solving skills. An approach called "behavior therapy" was also identified as a probably efficacious EBP (e.g., Kahn et al. 1990); its contents resembled CBT in most respects. Attention-Deficit/Hyperactivity Disorder. Pelham and Fabiano (2008), reviewing treatments for ADHD, identified three as well-established. One was behavioral parent training, in which parents learn techniques for effective behavior management (e.g., clear instructions, differential attention for desired vs. undesired behavior, use of praise and reward, time-out). A second was behavioral contingency management in classrooms, often carried out by school personnel. The third was intensive peer-focused behavioral interventions in recreational settings. A model program of this type is Pelham's Summer Treatment Program (Pelham et al. 2010), in which youngsters are immersed in sports, academic, and social skill-building activities, all within the context of a highly structured environment with clear behavioral contingencies; caregivers also participate, learning effective behavior management strategies for use at home. Importantly, Pelham and Fabiano did not find convincing support for CBT or non-behavioral treatments for ADHD youths.

Conduct-Related Problems and Disorders. In the review of treatments for conduct-related problems and disorders, Eyberg et al. (2008) identified 16 EBPs. These included behavioral parent training programs, some involving parent management training (Forgatch \& Patterson 2010; Kazdin 2010; Sanders \& Murphy-Brennan 2010) and some involving coaching during actual parent-child interactions (e.g., McMahon \& Forehand 2003; Zisser \& Eyberg 2010). The Incredible Years program (Webster-Stratton \& Reid 2010) includes a behaviorally-oriented video-guided series involving group sessions for parents (who view and discuss video clips 
illustrating effective and ineffective parenting strategies), and social skills and problem-solving training groups for children (ages 3-8); the parent and child programs have been shown to be beneficial separately and in combination. Other EBPs for conduct problems include behavioral and cognitive training to enhance anger management (Lochman et al. 2010), problem-solving skill (Kazdin 2010), and socially appropriate assertiveness (Huey \& Rank 1984), as well as a school-based program designed to reduce misbehavior by training youths to make accurate appraisals of self and social situations (Block 1978).

Finally, two of the EBPs identified by Eyberg et al. (2008) blend behavioral training for caregivers with strategies for building support within the youth's social system. These include the extensively-studied Multisystemic Therapy (MST; Henggeler \& Schaeffer 2010), developed originally for delinquent youths but later extended to other forms of dysfunction (e.g., sexual offending, suicidal behavior), and Multidimensional Treatment Foster Care (MTFC; Smith \& Chamberlain 2010), designed to support effective care for disruptive youths who have been placed in foster care. We later discuss MST and MTFC in greater detail.

Adolescent Substance Abuse. In their review of treatments for adolescent substance abuse, Waldron and Turner (2008) reported that interventions qualifying for EBP status have focused mainly on alcohol and marijuana use. The three EBPs they identified use a blend of behavioral methods, family systems work, and outreach to broader social systems. One of the EBPs, Functional Family Therapy (FFT; Alexander \& Parsons 1982), relies on core behavioral techniques and a family systems orientation to establish new patterns of family interaction, and therapists also work with extra-familial systems such as schools and probation offices to help ensure that benefits will generalize to the community. In addition, both individual and group CBT approaches (see Waldron \& Kaminer 2004) were identified as EBPs. In general, the CBT 
approaches focus on identifying and modifying distorted cognitions, plus training and practice in the behavioral coping skills needed to avoid substance use (e.g., coping with cravings, resisting social pressure to get high, and avoiding situations where substance use might be likely).

\section{Investigating the Strength, Causes, and Conditions of Effective Treatment}

Initially, researchers were most interested in identifying which psychotherapies work. Over time, however, interest has grown in how treatments work (i.e., through what processes), with whom they work, and under what conditions - questions to which we now turn.

Mediators and mechanisms of change. Understanding how a treatment works requires identifying mechanisms of change, the specific processes through which the treatment produces outcomes. A sound understanding of these mechanisms could help treatment developers strengthen the active ingredients of psychotherapy and minimize inactive components, thereby increasing treatment efficacy and cost-effectiveness (Kraemer et al. 2002). A first step in identifying change mechanisms is testing whether a particular variable is a mediator of treatment outcome in an RCT, that is, an intermediate variable evident during treatment that statistically accounts for the treatment-outcome relationship (Kraemer et al. 2002; Kazdin 2007).

Moderators of treatment outcome. Understanding with whom treatments work and under what conditions requires identifying moderators of treatment outcome. In an RCT, a moderator is a variable present prior to randomization that interacts with treatment condition, such that treatment effects depend on the level of the moderator (Kraemer et al. 2002). Identifying client characteristics that moderate treatment effects can reveal which client subgroups benefit most, and least, from various treatments. That information could help inform clinicians' efforts to identify the best-fit treatments for specific subgroups of patients (Kraemer et al. 2002).

\section{Mediators of Evidence-Based Psychotherapies}


To illustrate the search for mediators, we focus here on research testing two variables frequently studied in RCTs of youth EBPs — negative cognitions and parenting practices.

Negative cognitions. Negative cognitions have often been tested as candidate mediators of EBPs for internalizing disorders. This reflects the CBT theoretical model, which holds that symptoms can be reduced by changing cognitions that are unrealistically negative to those that are more positive, realistic, or adaptive.

Mediation tests within RCTs of CBT for youth depression have yielded mixed findings. Shifts to less pessimistic or more optimistic explanatory style have been found to mediate reductions in children's depressive symptoms (Jaycox et al. 1994; Yu \& Seligman 2002). Decreases in negative automatic thoughts and in dysfunctional core beliefs were significant mediators in some RCTs of CBT for adolescent depression, but not in others (Ackerson et al. 1998; Kaufman et al. 2005; Stice et al. 2010).

There have been fewer studies of mediation in CBT for youth anxiety. However, the studies have rather consistently found that decreases in anxious self-statements mediate reductions in anxiety symptoms in individual CBT (Kendall \& Treadwell 2007; Treadwell \& Kendall 1996) and group CBT (Lau et al. 2010).

Parenting practices. Parenting practices have often been tested as mediators of EBPs for externalizing problems. Most such EBPs aim to reduce youth problems partly through changing their caregivers' behaviors. These EBPs include behavioral parent training for mild conduct problems, MTFC and MST for severe conduct problems, MDFT for adolescent substance abuse, and behavioral treatment for ADHD.

Effective, consistent parental discipline has been found to mediate treatment effects on child conduct problems in RCTs of Parent Management Training - Oregon model (PMTO; Amlund 
Hagen et al. 2011) and anger control training (Lochman \& Wells 2002). For MTFC and MSTEBPs for adolescent offenders - mediators include not only caregivers' disciplinary practices, but also their ability to limit adolescents' association with deviant peers (Eddy \& Chamberlain 2000; Henggeler et al. 2009). Both caregiver supervision and positive caregiver-youth relationship were also significant mediators of MTFC (Eddy \& Chamberlain 2000), whereas caregiver supervision and caregiver-youth communication were not mediators of MST (Henggeler et al. 2009), and family cohesion was not a mediator of PMTO (Amlund Hagen et al. 2011).

For substance use problems, parent monitoring of adolescents' activities and peers has mediated the effects of MDFT, relative to peer group intervention, on adolescents' 12-month abstinence from substance use (Henderson et al. 2009). Intriguingly, mediation was found only when the outcome measured was the proportion of abstinent youths, but not when substance use frequency was the outcome; the authors concluded that parent monitoring may work when it prevents but not when it merely reduces substance use. Improved parent-adolescent relationship was not a mediator (Henderson et al. 2009).

Research on ADHD intervention in the Multimodal Treatment Study of Children with ADHD (MTA; MTA Cooperative Group 1999a) has identified parents' discipline practices as a mediator. In the MTA, medication (methylphenidate, Ritalin) only and combined medication and behavioral treatment (i.e., behavioral parent training, summer treatment program, and behavioral classroom management) outperformed behavioral treatment only, as well as community care. Curiously, discipline practices mediated treatment effects on children's social skills at school only when combination treatment — but not behavioral treatment or medication only—was 
compared to community care; other parenting practices (i.e., positive involvement, deficient monitoring) were not mediators (Hinshaw et al. 2000).

Discussion. The mediation research reviewed may shed some light on the EBPs' potential mechanisms of change. However, many questions remain. First, the small number of published mediation studies raises a concern that some of the mediators identified may represent chance findings, with many unpublished null findings languishing in file drawers. More replications are needed to confirm that even our best-supported candidate mediators, negative cognitions and parenting practices, are in fact robust mediators of EBP effects.

Second, findings were mixed; for example, negative cognitions did not consistently mediate CBT for depression, and parenting practices other than caregiver discipline did not consistently mediate EBPs for conduct problems. It is not clear whether these mixed findings reflect methodological artifacts, failure to differentiate related but distinct mediators, or true mediators that emerge only for certain conditions, age ranges, symptom severity, problem type, or protocol used; if so, this would represent what is called moderated mediation.

Third, we have not found clear evidence that change in any mediator preceded change in outcome; that temporal sequence is necessary for a mediator to be considered a true mechanism of change (Kazdin 2007). Many studies measured the mediator and outcome at pre- and posttreatment only, precluding efforts to clarify the temporal. For example, Hinshaw and colleagues (2000) could not determine whether combination treatment of methylphenidate plus behavioral therapy in the MTA (a) reduced parents' ineffective discipline, which in turn improved children's social skills; (b) improved children's social skills, which in turn led parents to reduce ineffective discipline; or (c) changed something else that caused improvement in both child social skills and parent discipline. Some (e.g., Henderson et al. 2009) have demonstrated that an 
earlier measurement of change in the mediator predicted a later measurement of change in outcome, but a mediator change that was measured earlier does not equate to a mediator change that occurred earlier. Frequent measurement of both candidate mediators and outcomes throughout and after treatment will be needed to determine which changes occur in which order (Kazdin 2007). When Stice and colleagues (2010) attempted such frequent measurement, they identified a paltry $8 \%$ of participants who met the temporal precedence criterion.

Fourth, a number of variables that have been conceptualized as change mechanisms have rarely been studied as mediators. Mediation studies of youth CBT have generally focused on cognitive variables, not behavioral variables such as engagement in pleasant activities or exposures, which are major components of CBT for depression and anxiety respectively (for exceptions, see Kaufman et al. 2005; Stice et al. 2010). In addition, mediation studies of EBPs for externalizing disorders have often neglected youth cognitive and behavioral variables such as hostile attributions, expectations of outcomes from aggressive behavior, locus of control, and school engagement, which have in fact been found to mediate treatment effects (Lochman \& Wells 2002; Leve \& Chamberlain 2007). Furthermore, few published studies have examined common factors (i.e., hypothesized change mechanisms shared by different psychotherapies, for different disorders, such as therapeutic alliance, hope, and readiness for change) as mediators in RCTs of youth EBPs. The therapeutic alliance, despite having been documented as a significant predictor of outcome in a meta-analysis of 38 studies of youth psychotherapy (McLeod 2011), has to our knowledge been subjected to a mediation test in only a single study (Kaufman et al. 2005). Moreover, we are not aware of any published mediation studies for several EBPs (e.g., EIBI, IPT-A, FFT), which each have theoretical change models that could be empirically tested in mediation studies. 
Fifth, at least two major multisite trials have found that combination treatment involving medication plus EBP was superior to medication alone and EBP alone-suggesting a need to study mediators and change mechanisms of combination treatments. As mentioned earlier, methylphenidate plus behavioral therapy was the most efficacious treatment in the MTA (MTA Cooperative Group, 1999a); in addition fluoxetine (Prozac) plus CBT was the most efficacious treatment in the Treatment for Adolescents with Depression Study (TADS; TADS Team 2004). Soberingly, in both these trials, both combination treatment and medication alone, but not EBP alone, outperformed the control group (community care and pill placebo respectively). In the MTA, both combination treatment and behavioral treatment alone improved parent discipline, but only for combination treatment was improved parent discipline related to improved child social skills and disruptive behavior (Hinshaw et al. 2000). In TADS, both combination treatment and CBT alone increased adolescents' readiness to change (specifically, their action orientation), and increases in readiness to change were associated with reductions in depressive symptoms (Lewis et al. 2009). However, pairwise contrasts of TADS treatment conditions were not conducted, thus it is unclear if the mediation effect reported by Lewis and colleagues applies to both combination treatment and CBT. The bottom line is that we simply do not know exactly how medication and EBP combine synergistically to bring about superior effects; we need to uncover the mediators and change mechanisms targeted by combination treatments.

\section{Moderators of Evidence-Based Psychotherapies}

Here, we review three categories of moderators - youth, caregiver, and methodological variables - found to be significant in meta-analyses of youth psychotherapies (encompassing multiple treatments, not exclusively EBPs) or in selected RCTs of youth EBPs. 
Youth characteristics. Youth symptom severity, comorbidity, demographics, and other pretreatment characteristics are often tested as potential moderators of EBPs. The specific youth variables that emerge as significant moderators have varied across EBP and disorder. We exclude here a number of youth characteristics for which evidence is extremely mixed (e.g., age and gender), but we offer two examples of factors for which findings have been intriguing.

Pretreatment symptom severity was identified as a significant moderator in several metaanalyses and RCTs. Higher severity was associated with larger treatment effects in two metaanalyses —one of psychotherapy for youth depression (Watanabe et al. 2007), the other of behavioral parent training for youth disruptive behavior (Lundahl et al. 2006) — possibly because there is more room for improvement among severely symptomatic youths. However, higher severity has also been linked to smaller effects of combination treatment and medication alone in the MTA (Hinshaw 2007). In addition, several RCTs have demonstrated that more symptomatic youths - unlike less symptomatic youths — derive greater benefit from more comprehensive, more intense, or family-focused therapies, compared to less comprehensive, less intense, or individual-focused therapies. For example, youths with more severe substance use and comorbidity at pretreatment benefited more from MDFT, which targets more risk factors including family interactions, than individual CBT or enhanced treatment as usual (Henderson et al. 2010). Moreover, among adolescents with anorexia nervosa, those with more severe overall symptoms or eating/weight-related obsessions and compulsions benefited more from a year-long course of family therapy compared to adolescent focused therapy of the same duration (Le Grange et al. 2012), and compared to a shorter 6-month course of family therapy (Lock et al. 2005). 
Comorbid diagnoses have moderated treatment effects among children with ADHD; for example, the subgroup with comorbid anxiety but no $\mathrm{CD} / \mathrm{ODD}$ responded equally well to behavioral treatment alone and medication alone, pointing towards behavior treatment as a potential first-line treatment for this subgroup of youths (Jensen et al. 2001; Hinshaw 2007).

Caregiver and family characteristics. Caregiver psychopathology, expressed emotion, and family demographics have been documented as significant moderators of EBPs. Here too, there is considerable variation across EBP and disorder in terms of which specific caregiver and family variables are treatment moderators, so again we offer just two examples of interesting findings. Critical, hostile, and emotionally overinvolved attitudes toward the patient by close family members — termed Expressed Emotion (EE; Hooley \& Parker (2006) — moderated family therapy outcomes for adolescent anorexia nervosa in one RCT but not in another. High EE was associated with better response to separated family therapy than to conjoint family therapy (Eisler et al. 2007).

However, EE did not moderate outcome in another RCT comparing family therapy to adolescent focused therapy (Le Grange et al. 2012). The authors surmised that separated family therapy may be especially suitable for adolescents from high EE families as it aims to reduce parent criticism while avoiding parent criticism during treatment sessions themselves. On the other hand, adolescent focused therapy aims to increase adolescent autonomy and does not thoroughly address the high levels of parent criticism in high EE families.

RCTs of several EBPs have documented family structure and socioeconomic status (SES) as moderators. Meta-analyses of behavioral parent training for disruptive behavior (Lundahl et al. 2006) and of behavioral and cognitive-behavioral interventions for ADHD (Corcoran \& Dattalo 2006) documented smaller treatment gains for youths from nonintact families; the first meta- 
analysis also documented smaller gains for low SES families. A plausible explanation is that single and low SES parents have less time and energy to attend therapy sessions and practice the skills learned during therapy in order to implement them effectively at home. Fortunately, there is also evidence that nonintact or low SES families benefit more from some versions of EBPs. Lundahl and colleagues' meta-analysis found that low SES families gained more from individual than group parent training, perhaps because the individual format allows greater attention to fitting the treatment to individual families' needs. In addition, adolescents with anorexia nervosa from nonintact families responded better to a year-long course than a 6-month course of family therapy (Lock et al. 2005), suggesting that adolescents from nonintact families may need longer treatment to derive meaningful benefit. These findings could potentially guide treatment decisions for youths based on their family demographics.

Method factors. The previous sections have focused on sample characteristics; we turn now to methodological factors that may moderate treatment outcome.

One of these involves the fact that different informants can differ markedly in their perspectives on the same youth's behavior, and on the outcome of treatment. Youth psychotherapy studies usually include outcome and process measures from multiple informants; these may include the youths themselves, caregivers, therapists, assessors not involved in providing treatment, siblings, peers, and teachers. Informant discrepancies are common and may be due to informant differences in both perspective and opportunities to observe the youth (De Los Reyes \& Kazdin 2005). Understandably, the presence, magnitude, and even direction of treatment effects can depend on the particular outcome measure used, and which informant completes that measure. Treatment effects were larger for youth-report than for parent-report measures in a meta-analysis of youth depression psychotherapy RCTs (Weisz et al. 2006b). 
Perhaps depressive symptoms such as sad mood are most salient to the youths experiencing them, and less so for outside observers, such that youths would be more sensitive to changes in their depressive symptoms than their parents would be. In a multisite trial of treatments for social phobia among children, parent-youth disagreement on youth symptoms at pretreatment were significantly associated with disagreement at posttreatment only among nonresponders to treatment (De Los Reyes et al. 2010).

Another aspect of informant report that may moderate treatment outcome is whether the informants are blind to treatment assignment. One relevant finding emerged from a recent metaanalysis of 52 RCTs comparing youth EBPs for an array of internalizing and externalizing disorders to usual clinical care (Weisz et al. 2013b — described below): the benefit of EBPs over usual care was significant only for youth-report and parent-report measures, but low and nonsignificant when outcomes were reported by the youths' therapists and teachers. Another recent meta-analysis obtained significant effects of behavioral treatments for ADHD that did not hold up when analyses were limited to outcome measures for which informants were likely to be blind to treatment condition (Sonuga-Barke et al. 2013; but note also that significant effects of dietary interventions for ADHD were evident with both blind and unblinded informants). Several explanations may account for better outcomes by unblinded informants: (a) informants who know about the treatment may be more invested in perceiving it as successful; (b) unblinded informants (e.g., youths and their caregivers) tend to also be those who have the most accurate and complete information about youth functioning; or (c) the measures most often taken via blinded informants are lacking in validity (e.g., observational ratings may not adequately sample the youth's behavior, or youths may behave in atypical ways when being observed). 
A third method factor warranting attention is the setting in which treatment takes place and in which youth behavior and outcomes are assessed. Numerous reviews (e.g., Weisz 2004) have noted that distinctive settings can carry distinctive constraints and can facilitate and inhibit very different behavior. The same youth may be very well-regulated within a highly-structured residential program but quite dysregulated at home or in school when the potent structure is no longer present. An effective behavioral parent training intervention may produce marked improvements in youth behavior at home that do not generalize to behavior in school. Prompted in part by such findings, the 2013 NICE guidelines for treatment of youth conduct problems in the UK recommend a multi-modal approach (see Scott et al. 2013, p. 33) in which treatment within the family is complemented by treatment focused on the school and other settings where youth behavior may be at issue. The challenge of cross-setting differences, and the closely-linked challenge of effect generalization, warrant careful research attention in the days ahead.

Therapist adherence and competence. We end this section on a puzzling note: findings across multiple studies have been quite mixed in regard to whether higher levels of therapist adherence to treatment manuals, and higher levels of measured therapist competence in the component skills, are actually associated with better or worse treatment outcomes, or no difference (see, e.g., Hogue et al., 2008; Webb et al., 2010; Weisz et al., 2012). Until there are more convergent findings (and perhaps refined measurement strategies will help), this important topic may remain shrouded in uncertainty, awaiting an empirical breakthrough.

\section{Adapting and Testing Psychotherapies for Diverse Populations}

In recent years, investigators have begun to examine the potency and reach of youth EBPs by evaluating their impact across a more and more diverse array of at-risk populations. We illustrate by focusing on four populations of special interest. 


\section{Ethnic Minority Populations}

The massive movement of population groups across national boundaries - due to violent conflict, repression, and pursuit of educational and employment opportunity - has created everhigher percentages of "minority youths" in countries around the world. In the U.S., for example, 2008 statistics showed that $43 \%$ of children and adolescents belonged to ethnic minority groups, and that percentage has continued to increase (Mather \& Pollard 2009). About $13 \%$ of minority

youths receive mental health services in the U.S. each year (Stagman \& Cooper 2010), but some reports on treatment process and outcome have not been encouraging. For example, a U.S. Department of Health and Human Services report (DHHS 2001) showed that only a small number of ethnic minorities had participated in psychotherapy RCTs, and these studies did not assess the efficacy of the treatment by ethnic status. Additionally, some research found that ethnic minority youths were significantly more likely than European-Americans to drop out of treatment (Kazdin \& Whitley 2003), and less likely to show significant clinical improvement when treated for depression (Weersing \& Weisz 2002a).

The results of a selective review conducted by Huey and Polo (2008) of youth treatment outcome studies from 1960 through 2006 was more promising. At least 75\% of participants in each study were ethnic minorities. Using the levels-of-support criteria we described earlier (see Silverman \& Hinshaw 2008), Huey and Polo found no "well-established" treatments for ethnic minority youths, but they identified a number of "probably efficacious" and "possibly efficacious" treatments for a range of psychological disorders including anxiety, ADHD, depression, conduct problems, substance use, posttraumatic stress, and suicide risk, with CBT as the best-documented EBP for anxiety disorders, depression, and trauma-related disorders. 
Adding a meta-analysis of 25 studies, Huey and Polo found a mean pre-post treatment effect size for ethnic minority youths averaging .44, just below a medium effect (Cohen 1988).

\section{Lower- and Middle-Income Countries and War-Exposed Youths}

Limited research on mental health has been conducted in lower- and middle-income countries (LAMICs; this constitutes 3-6\% of published mental health research (Saxena et al. 2006). This research gap is attributed to a range of factors, including limited mental health resources, a lack of trained mental health professionals, and child mortality concerns taking priority over morbidity (Patel et al. 2008).

Mental health efforts in LAMICs have primarily focused on the youths most in need of support: youths exposed to armed conflict in war-torn regions. These youths have experienced extreme psychosocial stressors such as military assault, sexual trauma, conscription as "child soldiers," and various consequences associated with involuntary displacement (e.g., refugee camps, a new and unfamiliar local language and culture, separation from family members). Thus, war-exposed youths are at heightened risk for substance use, depression, anxiety, conduct problems, posttraumatic stress, and suicidal ideation (Shaw 2003; Lustig et al. 2004).

The modest research conducted to date on treatment for war-exposed youths is somewhat encouraging. Bolton et al. 2007) assessed a group interpersonal therapy (IPT) intervention for depression among adolescent Ugandan war refugees. Compared to a creative play program and waitlist control, IPT was significantly more effective in reducing depression symptoms (but not anxiety or conduct problems) for girls; symptom reductions occurred for boys but were nonsignificant. Dybdahl (2001) assessed a psychosocial treatment focused on parent traumaprocessing and improving mother-youth interactions for mother-youth refugee dyads internally displaced within Bosnia. The treatment group showed significantly greater improvement in 
mothers' PTSD symptoms and youths' psychosocial functioning (youths' PSTD symptoms were not explicitly assessed), as compared to a control group.

Large-scale RCTs have also been conducted as school-based interventions. Trauma and grief component therapy (TGCT) has been widely disseminated in Bosnia as part of a UNICEF mental health program. Layne et al. 2008) assessed the effectiveness of school-based TGCT for Bosnian adolescents with PTSD, depression, or grief symptoms. Students participated in one of two treatment conditions consisting of components of TGCT: trauma- and grief-centered therapy with psychoeducation and social skills training (treatment condition), or psychoeducation with social skills training (active comparison group). Both TGCT groups showed a significant reduction in PTSD and depression symptoms, but only the treatment condition showed a significant reduction in grief symptoms. Jordans et al. 2010) assessed a classroom-based intervention in civil war-affected Nepal designed to improve general well-being and psychosocial functioning among adolescents. The intervention consisted of CBT, narrative exposure techniques, and structured play. Compared to a wait-list control, the treatment group experienced significantly greater improvements in overall functioning, general psychological functioning, and greater reduction in depression and anxiety symptoms. PTSD symptoms decreased and feelings of hope increased to a similarly significant degree in both groups. Tol et al. (2008) tested a similar CBT intervention for PTSD symptoms among youths in Indonesia exposed to political violence, and the treatment group showed a significantly greater reduction in PTSD symptoms (but not depression or anxiety symptoms) compared to a waitlist control. These studies show promising initial dissemination efforts of evidence-based treatments to war-exposed youths even in remote and limited-resource regions. Perhaps these early findings will be a springboard for work with other LAMIC populations greatly in need of support. 


\section{Youths Offenders in the Juvenile and Criminal Justice System}

Previous studies have found high prevalence of psychological disorders in incarcerated youth offenders. Teplin and colleagues (2006) found that the most common disorders included conduct-related, substance use, and affective disorders, with comorbidity quite substantial. The primary objective of the juvenile justice system is rehabilitation, and treatment for mental health problems is widely viewed as essential. However, there is little empirical support for many of the interventions used, and some may be ineffective or harmful. Lipsey \& Wilson (1998) conducted a meta-analysis of 200 intervention studies for juvenile offenders and found a small effect for reduction in rate of reoffending (average effect size of 0.1).

The Blueprints for Violence Prevention project of the Center for the Study and Prevention of Violence, at the University of Colorado, has evaluated over 900 programs designed to rehabilitate juvenile delinquents (Mihalic et al. 2002). Evidence in support of most of these programs (e.g., boot camp, summer job programs) was found to be either disappointing or entirely lacking. Arguably the strongest evidence supports Multisystemic Therapy (MST; Glisson et al. 2010; Henggeler \& Schaeffer 2010; Schoenwald 2010), an intensive intervention targeting multiple systems in youths' lives, including family support and relationships, neighborhood and peer networks, and their teachers and school. Across multiple trials, MST has been shown to significantly reduce rate of reoffending, improve parenting skills and family relations, enhance positive social networks and prosocial activities, and improve grades and vocational skills. MST has been shown to be more effective than common "usual care" alternatives, including probation services, child-welfare systems, and individual outpatient therapy (Weisz et al. 2012). Unfortunately, practices found to be ineffective, or even harmful, continue to be widely used. 


\section{Youths Cared for in Child Protection Programs}

Child protection and child welfare systems are typically designed to balance the value of family cohesion against the need to protect children from maltreatment. These systems may remove children from their home or assist parents in child-rearing. The youths involved often have significant mental health needs, sometimes related to family stress or maltreatment they experienced, sometimes to the process of foster placement (often a series of placements), and sometimes to diverse factors not all of which can be identified. Unsurprisingly, admittance into the child protection system is a common precursor to mental health services (see Garland et al. 1996; Garland et al. 2001a; Garland et al. 2001b).

Successful interventions for these youngsters often engage multiple systems in the youths' lives. One example, Multisystemic Therapy-Child for Child Abuse and Neglect (MSTCAN), consists of the standard components of MST in addition to safety planning, parental anger management, and family problem-solving and communication training. In one RCT, MST-CAN was shown to be more effective than enhanced outpatient treatment in reducing youth psychopathology symptoms and placement changes, and improving parent functioning, including reduced maltreatment (Swenson et al. 2010).

Another successful approach is Multidimensional Treatment Foster Care (MTFC; Smith \& Chamberlain 2010). MTFC involves working with youths, biological parents, and foster parents using a multilevel intervention implemented in family, community, and school settings. Each youth is placed in a foster home for 6 to 9 months, where an intensive individualized behavior management program is implemented. Parents attend family therapy, and learn parenting strategies including limit-setting and positive reinforcement of prosocial behaviors. MTFC has been shown to be more effective in reducing youth delinquent behavior than standard 
group care (Chamberlain \& Reid 1998; Chamberlain et al. 2007). Other MTFC trials have found reductions in severe youth mental health problems (Chamberlain et al. 1992).

Youths in the child welfare system with histories of maltreatment who are at increased risk for PTSD may need trauma-focused mental health services. The most thoroughly-tested of these is Trauma-Focused Cognitive Behavioral Therapy (TF-CBT; Cohen et al. 2010). TF-CBT integrates traditional CBT with trauma-specific methods, including narrative exposure therapy, safety planning, and graduated exposure to trauma-related cues. RCTs have shown TF-CBT to be more effective in reducing PTSD symptoms than waitlist control, "supportive therapy," and "child-centered therapy" (see review in Cohen et al. 2010).

\section{Putting Science into Practice: EBPs and the Clinical Practice of Youth Mental Health Care}

A central goal for so many who develop treatments and do treatment outcome research is to improve mental health care for the many girls and boys who struggle with problems and disorders in their everyday lives. Because most of these youngsters will never participate in a treatment trial, prospects for them to benefit from the research will depend on the extent to which that research informs and improves everyday clinical practice. This means that the interventions developed and tested will need to be appropriate for and adopted by clinical practice, and when used in practice will actually lead to improved outcomes. We now take stock of how well youth treatment research is doing in relation to these objectives.

\section{Dissemination and Implementation of Evidence-Based Practices}

The objectives are closely related to the subfield now known as dissemination and implementation science. Dissemination has been defined (National Institute of Mental Health [NIMH] 2009) as: 
“....the targeted distribution of information and intervention materials to a specific public health or clinical practice audience. The intent is to spread knowledge and the associated evidence-based interventions.”

Implementation has been defined (NIMH 2009) as"

"the use of strategies to adopt and integrate evidence-based health interventions and change practice patterns within specific settings."

What grade should we give youth psychotherapy research on the complementary tasks of dissemination and implementation? On the one hand, there is now extensive publicity for EBPs, within prominent journals and through government entities in many nations. On the other hand, despite all the publicity, most of the tested treatments are still used mainly in treatment trials; most have not made significant inroads into everyday clinical care. Studies in North America show very low levels of EBP use in everyday practice (see Weisz et al. 2009; Brookman-Frazee et al. 2010; Garland et al. 2010; Southam-Gerow et al. 2010). Complementing these reports, a UK survey published in 2006 by the Association for Child and Adolescent Mental Health (see http://www.acamh.org.uk/POOLED/articles/bf_eventart/view.asp?Q=bf_eventart_213513) found that CBT was the dominant approach of only $20 \%$ of practitioner respondents, despite very substantial government pressure favoring CBT (e.g., via the NICE guidelines). While the data from North America and the UK certainly do not cover all practice or training sites, they do suggest that EBPs are not making their way into training or practice very quickly. Why not? Why wouldn't professionals who chose careers to help young people be eager to adopt practices that have been tested and shown to work?

Goodness-of-Fit Issues in the Design of Evidence-Based Practices 
One answer may relate to the design of the tested practices. Research in clinical care settings and with practitioner partners (e.g., Weisz 2004; Weisz et al. 2005b, 2013a) has raised significant design concerns. One is the fact that most of the EBPs (see Table 1) are designed for single problems or disorders (or homogeneous clusters - e.g., a few depressive disorders). For practitioners, whose caseloads tend to be broad and heterogeneous, with an array of disorders and significant comorbidity within cases, learning one or two single-disorder EBPs may not be seen as useful enough to warrant the time and cost. An additional concern is that EBPs tend to ask a lot of therapists-learning a detailed manual, much more advance preparation for each session than may seem feasible in everyday practice, working from an agenda rather than letting the session flow freely, and devising ways to make the manualized content engaging and motivating for the youth. None of these challenges is insurmountable, but each may require attention in efforts to disseminate EBPs, and even in the very development and testing of EBPs, a topic to which we turn next.

\section{Context of EBP Development and Testing versus Conditions of Everyday Practice}

The structure and content of EBPs, and the degree to which they fit the conditions of everyday practice, may have a lot to do with the conditions under which they are created and tested. In general, these conditions have differed markedly from the conditions of actual clinical practice (Weisz et al. 2005a; Weisz \& Gray 2008). This difference may impose certain limits on prospects for extrapolating from research findings to treatment in youth clinical service settings. The difference may also have slowed the pace of dissemination and implementation, to some degree, in part by raising practitioner concern over whether these treatments that look promising in research are actually appropriate for their use in real-world clinical practice. 
The research vs. clinical practice differences take multiple forms, related to the therapy participants, the treatment settings, and even the broader policy and fiscal context. Youths treated in clinical practice settings may differ from those recruited for RCTs in diverse ways, including severity and family adversity (Hammen et al. 1999; Southam-Gerow et al. 2003). Clinicians employed in clinics differ from research-employee therapists in background and training, daily working conditions, incentive structure, and professional goals (see Weisz \& Addis 2006; Palinkas et al. 2008). In addition, clinical practice and research organizations differ in multiple ways relevant to implementation of EBPs; for example, in practice settings, time, productivity, financial, and other work pressures can make it hard to allocate time to the training and ongoing case consultation needed for successful implementation of EBPs (Weisz \& Addis 2006). Such differences — encompassing the patients, therapists, and settings of therapy—illustrate the stark gap between the world of clinical research and that of clinical practice.

\section{(Un)Representiveness of Most Research on Evidence-Based Youth Psychotherapies}

In principle, that gap might be addressed by basing much of the research that leads to EBPs in representative clinical practice contexts. In fact, though, such research has been rare, to date. The problem is illustrated by Table 2, which summarizes relevant details of 461 youth psychotherapy RCTs from the 1960s through 2009. As shown in the table, the large majority of trials used (a) recruited (not clinically referred) samples, (b) therapists who were research employees (not practicing clinicians), and (b) university lab or other non-practice settings. The table also shows that representativeness has increased somewhat in recent decades, but only $2.1 \%$ of all the studies, and only $4.5 \%$ of studies in the most recent decade, focused on clinically referred clients, treated by practitioners, in clinical practice settings (see also Weisz et al. 2005a, for a similar analysis and similar conclusions). What seems clear is that most of the research that 
leads to the establishment of EBPs has been carried out in contexts quite different from the world of clinical practice for which the EBPs are ultimately intended. One result may be a collection of treatments that are a less than perfect fit to that clinical practice world.

\section{Do EBPs Actually Produce Better Outcomes than Usual Clinical Care?}

Concerns about the fit between EBPs and usual clinical practice lead quite naturally to a critical question: Do EBPs produce better outcomes than the treatments youths would otherwise receive through usual clinical care? In some respects, this is the most basic question many in clinical practice might ask. Given the very substantial funding and time required to build competence in a typical manual-guided EBP, it is reasonable for practitioners, clinic directors, policy makers, and funders to ask whether shifting from usual treatment practices to EBPs will in fact lead to better youth outcomes than current practices do. Youth treatment research has not emphasized the kinds of studies that could answer that question-i.e., RCTs in which youths with significant mental health problems or disorders are randomly assigned to a specific EBP or to usual clinical care. However, a limited pool of such studies does exist, and we turn to those studies next.

Our search for relevant studies has led to two meta-analyses focused on methodologicallyacceptable studies that have directly compared usual care to youth psychotherapies that have been formally classified as EBPs. In these two meta-analyses (32 studies in Weisz et al., 2006b; 52 studies in Weisz et al., 2013b) studies spanned the years 1973-2010, and youth ages ranged from 3-18 years. Most of the EBPs were specific behavioral or CBT interventions, and about a fourth were systems-oriented approaches such as MST. On average, the EBPs did outperform usual care. However, the mean post-treatment effect size was only .30 and .29, in the first and second meta-analysis respectively, falling between conventional cutoffs for 'small' and 
'medium,' and markedly lower than mean effects found in prior meta-analyses (see Figure 1). To put the findings into context, a mean effect size of .29 to .30 translates into a probability of only $58 \%$ that a randomly selected youth receiving an EBP would be better off after treatment than a randomly selected youth receiving usual care. Also worrisome: in the 2013 meta-analysis, there was no significant advantage of the EBPs with two key subgroups: clinically-referred samples and youths in samples severe enough to be clinically diagnosed. A more pessimistic picture was presented in Spielmans and colleagues' (2010) reanalysis of the Weisz et al. (2006b) meta-analysis. These authors controlled for factors they believed might bias study findings in favor of EBPs, and concluded that EBPs did not significantly outperform usual care. Readers might also find some support in both the Spielmans et al. and Weisz et al. meta-analyses for the notion that effect sizes shrink when EBPs are tested by independent investigators with no involvement by the program developers (see Eisner, 2009). One conclusion on which all the meta-analysts would likely agree is that outcomes have been highly variable across studies, with a number of studies showing usual care outcomes to be similar or superior to EBPs outcomes. This variability is shown in Figure 2, for the Weisz et al. (2013b) meta-analysis study sample.

\section{Strategies for Strengthening Youth Psychotherapies and Intervention Science}

The findings reviewed thus far point to a lively field with committed researchers actively searching for beneficial youth treatments. The findings also suggest strategies for building stronger treatments and improving the evidence base on youth psychotherapy.

\section{Making Intervention Research Look More Like Practice}

A significant limitation of the evidence base, shown in Table 2, is that intervention research shows relatively low relevance to actual clinical practice. We have argued (e.g., Weisz 2004; Weisz \& Gray 2008) for a deployment-focused model within which interventions are developed 
and tested, as soon as feasible, with the kinds of therapists and clients, and in the kinds of settings, for which the interventions are ultimately intended. If this model were adopted, the form of research known as efficacy testing (e.g., with recruited samples treated by research-employee therapists under experimenter-designed conditions) would be only a brief initial phase in intervention development, used to establish potential for benefit, and effectiveness testing under clinically representative conditions would be the dominant research approach. This in turn would provide a more direct path than now exists between research on youth psychotherapy and effective applications of the products of that research within everyday youth clinical care.

\section{Tapping the Heuristic Potential of Usual Care}

Speaking of usual care, our evidence suggests that it may have something to teach us. Figure 2 revealed that some forms of usual care produced outcomes comparable to or better than the outcomes of EBPs, suggesting that some treatments arising from everyday practice may deserve to be documented in written protocols and tested in their own right. For this to be feasible, however, investigators will need to stop treating usual care as a mere 'control condition' and think of it instead as source of ideas and methods. Weisz et al. (2006a) noted that the studies in their meta-analysis generally provided excellent documentation of EBP content but very poor documentation of the usual care conditions to which the EBPs were compared, leaving it unclear what 'usual care' actually consisted of. This problem needs to be solved if we are to maximize the hypothesis-generating potential of EBP vs. usual care research. Methods are now available for documenting usual care contents through a standardized clinician checklist (Weersing et al. 2002) and through a system for observer coding of intervention sessions (McLeod \& Weisz 2005, 2010). The checklist has been used to describe usual care for a variety of youth problems and conditions (Weersing et al. 2002), and the observational coding system has been used to 
document characteristics of usual care in treatment of youth disruptive disorders (Brookman-

Frazee et al. 2010; Garland et al. 2010) and depressive and anxiety disorders (Weisz et al. 2009;

Southam-Gerow et al. 2010). In the next generation of studies, these and related methods could be used to characterize those patterns of usual care that show evidence of benefit.

\section{Taking Risks and Learning from Both Failure and Harm}

The need for more potent treatments argues for testing creative ideas and daring ventureshigh-risk/high-gain efforts toward breakthrough innovations in treatment. However, taking risks with daring innovations virtually ensures some failures. Each failure may add valuable information on treatments that don't work - an important complement to learning what does work; both form the empirical tapestry of our science. As any meta-analyst would note, reporting failures is critical to a balanced picture; woe be the science in which only positive results are published. Failure can also be a springboard for treatment refinement and improvement, as when group intervention with antisocial youths was found to produce iatrogenic "peer contagion" or "deviancy training" effects, suggesting the need for a different approach (Dishion \& Dodge, 2005), or when the discovery that a particular implementation of parent training didn't work suggested innovative enrichment strategies (Scott \& Dadds, 2009). Evidence indicates that even evidence-based treatments can have harmful effects if poorly executed (Scott, 2013), thus highlighting the need for rigorous implementation. Treatment failure or weak effects can also prompt useful tests of dose-augmenting strategies, such as boosters and relapse prevention, in programs for both internalizing and externalizing problems (see e.g., Clarke \& DeBar, 2010; Tolan et al. 2009).

\section{Restructuring Evidence-Based Psychotherapies to Fit Clinical Practice Needs}


We suggested earlier that many EBPs are designed in ways that may not match up well with the clients, clinicians, and settings of everyday clinical care. For example, most EBPs are built for a single disorder or a small homogeneous cluster, whereas most clinicians carry diverse caseloads, such that one EBP for one disorder may fit only a tiny portion of their clientele. Single-disorder EBPs may also not mesh well with the fact that most referred clients have comorbid conditions, and that the most pressing problems that need attention can shift during treatment, rendering the single focus with which treatment began no longer so relevant. One approach to addressing the problems of diverse caseload, client comorbidity, and flux in treatment is to build integrative treatments that combine intervention components from multiple EBPs, encompassing multiple different problems and disorders. As an example, Chorpita and Weisz (2009) developed a modular protocol for child and adolescent disorders and problems involving anxiety, depression, and disruptive conduct. In a recent randomized effectiveness trial, this protocol produced significantly better clinical outcomes than (a) usual outpatient care and (b) standard single-disorder protocols (Weisz et al. 2012). Such integrative treatments may not only improve effectiveness but boost efficiency, reducing the need to do multiple trainings and to navigate multiple single-disorder protocols.

\section{Improving Care through Monitoring and Feedback}

Another development that may improve clinical care and outcomes is the design and regular use of information systems that provide feedback on client response. Such a system was used with the modular protocol for youth, described in the previous paragraph (Chorpita \& Weisz 2009); the system provided weekly feedback on youth progress throughout treatment using two brief, psychometrically sound measures (Chorpita et al. 2010; Weisz et al. 2011), displayed on a "client dashboard" within an accessible web-based system. Recent research indicates that 
providing such client progress feedback to clinicians, even in the absence of EBPs, may improve therapy success and reduce deterioration among adults (Lambert et al. 2002; Anker et al. 2009) and improve therapeutic relationships and outcomes among youths (Stein et al. 2010; Bickman et al. 2011).

\section{Enriching Our Understanding of What Makes Treatments Work}

Although we listed forty youth EBPs at the beginning of this chapter, we have found very few replicated mediators of youth EBPs, and there is literally no evidence on whether any of these actually preceded changes in outcome. In fact, reviews (Weersing \& Weisz 2002b; Chu \& Harrison 2007) indicate that potential mediators of youth EBPs are often measured but not subjected to formal mediation testing. Consequently, we know a lot about which psychotherapies work for youth, but we know remarkably little about how these treatments work. This is unfortunate because understanding change mechanisms can help us build potent models of behavior change that can, in turn inform development of powerful treatments (Kazdin \& Nock 2003). Identifying true mechanisms of change is resource-intensive, requiring frequent measurement of multiple candidate mediators and outcomes as well as experimental manipulation of mediators (Kazdin 2007). But the effort may well be warranted, especially as we now have particularly promising statistical methods (e.g., bias-corrected bootstrapping, joint significance testing, and PRODCLIN asymmetric confidence-intervals testing; see Fritz \& MacKinnon 2007) for drawing strong inferences about the active ingredients that make treatments work.

\section{Building Efficient and Accessible Delivery Methods and Models}

To increase accessibility and cost-effectiveness of treatment, some researchers have deviated from traditional in-person talk therapy. In fact, Kazdin and Blase (2011) have proposed a 
"portfolio" of treatment delivery methods to increase the public-health impact of psychotherapies. These methods may include (but certainly not be limited to) self-help books that impart EBPrelated skills (e.g., Forehand \& Long 2010), treatments enlivened through video materials (e.g., Webster-Stratton \& Reid 2010), therapies delivered by paraprofessionals (e.g., Yu \& Seligman 2002), and therapies embedded within everyday settings such as summer camps (e.g., Pelham et al. 2010). Such approaches may increase the uptake and spread of EBPs by reducing cost, travel time, logistical barriers, and even the stigma associated with traditional treatment methods, and by boosting engagement for many.

Another approach noted by Kazdin and Blase (2011) deserves special attention: the use of computer and internet technology (see Enock \& McNally, 2013) for a review and relevant model). As one example, Kendall and Khanna (2008) developed a computer-assisted treatment for youth anxiety disorders, Camp Cope-A-Lot, based on the Coping Cat program. The youngster interacts with animated figures on the monitor to complete session activities, and the therapist coaches the patient through the program. Merry and colleagues (2012) developed SPARX, a computer-based treatment for youth depression. SPARX is a video game that conveys CBT skills such as problem solving, cognitive restructuring, and relaxation. Initial evidence suggests that both Camp Cope-ALot (Kendall et al. 2011) and SPARX (Merry et al. 2012) may be as effective as standard individual CBT.

Technology-assisted interventions for conduct problems also appear promising. As one example, Enebrink and colleagues (2012) developed an internet-based version of parent management training that includes illustrations and video clips illustrating positive parenting strategies; this internet PMT significantly outperformed a waitlist control condition in reducing 
youth conduct problems, increasing youth prosocial behavior, and enhancing positive parenting. Thus, early evidence suggests that some efficient, low-cost technology-assisted approaches to treatment may be no less effective than costly traditional methods. With further advances in technology, expansion of these alternatives seems quite likely and potentially promising.

Ideally, efforts to improve efficiency and cost-effectiveness would be guided by precise methods of calculating the cost of each approach. However, the calculations for, say, implementing a traditional manualized EBP may include the expense of initial training, ongoing expert supervision, therapist time preparing for sessions, therapist billing time foregone (during trainings and supervision), clinic administrative time, and other factors for many of which precise costs may be difficult to nail down. This may make it difficult to nail down the benchmark cost of standard approaches, and thus how much costs have been reduced by the use of technology and the other innovations noted in this section. Future collaboration with our health care economist colleagues could sharpen our ability to calculate costs precisely and thus assess whether, and if so how much, cost-effectiveness is boosted by these new approaches.

\section{Summary and Concluding Comment}

Research on youth psychotherapy has achieved remarkable gains in only five decades. There is now a rich treasury of tested treatments for an array of youth problems and disorders, an evolving process for evaluating the strength of the evidence for each of these, a vibrant intervention science focused on understanding how these treatments work and under what conditions, and a groundswell of interest and enterprise focused on strategies for making these treatments more and more accessible to more and more youths and families. There is a great deal of room for improvement in the interventions themselves and in the science that guides their 
development and refinement. Nonetheless, fifty years of research has produced a remarkable body of work and a remarkable launch for the scientific study of youth psychotherapy. 


\section{References}

Ackerson, J. et al. (1998) Cognitive bibliotherapy for mild and moderate adolescent depressive symptomatology. Journal of Consulting and Clinical Psychology 66, 685-690.

Alexander, J.F. \& Parsons, B.V. (1982) Functional Family Therapy: Principles and Procedures Brooks/Cole, Monterey, CA.

Amlund Hagen, K. et al. (2011) Treatment outcomes and mediators of parent management training: A one-year follow-up of children with conduct problems. Journal of Clinical Child and Adolescent Psychology 40, 165-178.

Anker, M.G. et al. (2009) Using client feedback to improve couple therapy outcomes: A randomized clinical trial in a naturalistic setting. Journal of Consulting and Clinical Psychology 77, 693-704.

Barlow, D.H. et al. (2009) Single Case Experimental Designs: Strategies For Studying Behavior Change Allyn \& Bacon, Boston, MA.

Barrett, P.M. et al. (2008) Evidence-based psychosocial treatments for child and adolescent obsessive-compulsive disorders. Journal of Child and Adolescent Clinical Psychology 37, $131-155$.

Beidel, D.C. et al. (2000) Behavioral treatment of childhood social phobia. Journal of Consulting and Clinical Psychology 68, 1072-1080.

Bickman, L. et al. (2011) Effects of routine feedback to clinicians on mental health outcomes of youths: Results of a randomized trial. Psychiatric Services 62, 1423-1429.

Block, J. (1978) Effects of a rational-emotive mental health program on poorly achieving, disruptive high school students. Journal of Counseling Psychology 25, 61-65. 
Bolton, P. et al. (2007) Interventions for depression symptoms among adolescent survivors of war and displacement in Northern Uganda: A randomized controlled trial. Journal of the American Medical Association 298, 519-527.

Brookman-Frazee, L. et al. (2010) Factors associated with use of evidence-based practice strategies in usual care youth psychotherapy. Administration \& Policy in Mental Health \& Mental Health Services Research 37, 254-269.

Campbell, J.M. (2003) Efficacy of behavioral interventions for reducing problem behavior in persons with autism: A quantitative synthesis of single-subject research. Research in Developmental Disabilities 24, 120-138.

Casey, R.J. \& Berman, J.S. (1985) The outcome of psychotherapy with children. Psychological Bulletin 98, 388-400.

Chamberlain, P. \& Reid, J.B. (1998) Comparison of two community alternatives to incarceration for chronic juvenile offenders. Journal of Consulting and Clinical Psychology 66, 624-633.

Chamberlain, P. et al. (1992) Enhanced services and stipends for foster parents: Effects on retention rates and outcomes for children. Child Welfare 5, 387-401.

Chamberlain, P. et al. (2007) Multidimensional Treatment Foster Care for girls in the juvenile justice system: A 2-year follow-up of a randomized clinical trial. Journal of Consulting and Clinical Psychology 75, 187-193.

Chambless, D.L. et al. (1998) Update on empirically validated therapies II. Clinical Psychologist $51,3-16$.

Chen, C., \& Ma, H. (2007) Effects of treatment on disruptive behaviors: A quantitative synthesis of single-subject researches using the PEM approach. Behavior Analyst Today 8, 380-397. 
Chorpita, B.F. \& Weisz, J.R. (2009) Modular Approach to Therapy for Children with Anxiety, Depression, Trauma, or Conduct Problems (MATCH-ADC) PracticeWise, Satellite Beach, FL.

Chorpita, B.F. et al. (2010) Evaluation of the brief problem checklist: Child and caregiver interviews to measure clinical progress. Journal of Consulting and Clinical Psychology 78, $526-536$.

Chu, B.C. \& Harrison, T.L. (2007) Disorder-specific effects of CBT for anxious and depressed youth: A meta-analysis of candidate mediators of change. Clinical Child and Family Psychology Review 20, 352-372.

Clarke, G.N., \& DeBar, L. (2010) Group cognitive-behavioral treatment for adolescent depression. In: Evidence-Based Psychotherapies for Children and Adolescents. (eds J.R. Weisz \& A.E. Kazdin), 2nd edn, pp. 110-125. Guilford Press, New York, NY.

Cohen, J. (1988) Statistical power analysis for the behavioral sciences Erlbaum, Hillsdale, NJ.

Cohen, J.A. et al. (2010) Trauma-focused cognitive-behavioral therapy for traumatized children. In: Evidence-Based Psychotherapies for Children and Adolescents. (eds J.R. Weisz \& A.E. Kazdin), 2nd edn, pp. 295-311. Guilford Press, New York, NY.

Corcoran, J. \& Dattalo, P. (2006) Parent involvement in treatment for ADHD: A meta-analysis of the published studies. Research on Social Work Practice 16, 561-570.

David-Ferdon, C., \& Kaslow, N.J. (2008) Evidence-based psychosocial treatments for child and adolescent depression. Journal of Child and Adolescent Clinical Psychology 37, 62-104.

De Los Reyes, A., \& Kazdin, A.E. (2005) Informant discrepancies in the assessment of childhood psychopathology: A critical review, theoretical framework, and recommendations for further study. Psychological Bulletin 131, 483-509. 
De Los Reyes, A. et al. (2010) The relations among measurements of informant discrepancies within a multisite trial of treatments for childhood social phobia. Journal of Abnormal Child Psychology 38, 395-404.

Dishion, T.J., \& Dodge, K.A. (2005) Peer contagion in interventions for children and adolescents: Moving toward an understanding of the ecology and dynamics of change. Journal of Abnormal Child Psychology 33, 395-400.

Dybdahl, R. (2001) Children and mothers in war: An outcome study of a psychosocial intervention program. Child Development 71, 1214-1230.

Eddy, J.M., \& Chamberlain, P. (2000) Family management and deviant peer association as mediators of the impact of treatment condition on youth antisocial behavior. Journal of Consulting and Clinical Psychology 68, 857-863.

Eisler, I. et al. (2007) A randomized controlled treatment trial of two forms of family therapy in adolescent anorexia nervosa: A 5-year follow-up. Journal of Child Psychology and Psychiatry 48, 552-560.

Eisner, M. (2009) No effects in independent prevention trials: Can we reject the cynical view? Journal of Experimental Criminology 5, 163-183.

Enebrink, P. et al. (2012) Internet-based parent management training: A randomized controlled study. Behaviour Research and Therapy 50, 240-249.

Enock, P.M., \& McNally, R.J. (2013) How mobile apps and other web-based interventions can transform psychological treatment and the treatment development cycle. The Behavior Therapist 36, 56-63.

Eyberg, S.M. et al. (2008) Evidence-based psychosocial treatments for children and adolescents with disruptive behavior. Journal of Child and Adolescent Clinical Psychology 37, 215-237. 
Eysenck, H.J. (1952) The effects of psychotherapy: An evaluation. Journal of Consulting Psychology 16, 319-324.

Forehand, R., \& Long, N. (2010) Parenting the Strong-Willed Child, 3rd edn. McGraw-Hill, New York, NY.

Forgatch, M.S. \& Patterson, G.R. (2010) Parent management training—Oregon model: An intervention for antisocial behavior in children and adolescents. In: Evidence-Based Psychotherapies for Children and Adolescents. (eds J.R. Weisz \& A.E. Kazdin), 2nd edn, pp. 159-177. Guilford Press, New York, NY.

Fritz, M.S. \& MacKinnon, D.P. (2007) Required sample size to detect the mediated effect. Psychological Science 18, 233-239.

Garland, A.F. et al. (1996) Type of maltreatment as a predictor of mental health service use for children in foster care. Child Abuse and Neglect 20, 675-688.

Garland, A.F. et al. (2001a) Multi-sector complexity of systems of care for youth with mental health needs. Children's Services: Social Policy, Research, and Practice 4, 123-140.

Garland, A.F. et al. (2001b) Prevalence of psychiatric disorders in youths across five sectors of care. Journal of the American Academy of Child and Adolescent Psychiatry 40, 409-418.

Garland, A.F. et al. (2010) Mental health care for children with disruptive behavior problems: A view inside therapists' offices. Psychiatric Services 61, 788-795.

Glisson, C. et al. (2010) Randomized trial of MST and ARC in a two-level evidence-based treatment implementation strategy. Journal of Consulting Psychology 78, 537-550.

Hammen, C. et al. (1999) The context of depression in clinic-referred youth: Neglected areas in treatment. Journal of the American Academy of Child and Adolescent Psychiatry 38, 64-71. 
Henderson, C.E et al. (2009) Parenting practices as mediators of treatment effects in an earlyintervention trial of multidimensional family therapy. American Journal of Drug and Alcohol Abuse 35, 220-226.

Henggeler, S.W. \& Schaeffer, C. (2010) Treating serious antisocial behavior using Multisystemic Therapy. In: Evidence-Based Psychotherapies for Children and Adolescents. (eds J.R. Weisz \& A.E. Kazdin), 2nd edn, pp. 259-276. Guilford Press, New York, NY.

Henggeler, S.W. et al. (2009) Mediators of change for multisystemic therapy with juvenile sexual offenders. Journal of Consulting and Clinical Psychology 77, 451-462.

Hinshaw, S.P. (2007) Moderators and mediators of treatment outcome for youth outcome for youth with ADHD: Understanding for whom and how interventions work. Journal of Pediatric Psychology 32, 664-675.

Hinshaw, S.P. et al. (2000) Family processes and treatment outcome in the MTA: Negative/ineffective parenting practices in relation to multimodal treatment. Journal of Abnormal Child Psychology 28, 555-568.

Hogue, A. et al. (2008) Treatment adherence, competence, and outcome in individual and family therapy for adolescent behavior problems. Journal of Consulting and Clinical Psychology 76, $544-555$.

Hooley, J.M. \& Parker, H.A. (2006) Measuring expressed emotion: An evaluation of the shortcuts. Journal of Family Psychology 20, 386-396.

Huey, S.J. Jr. \& Polo, A.J. (2008) Evidence-based psychosocial treatments for ethnic minority youth. Journal of Clinical Child and Adolescent Psychology 37, 262-301.

Huey, W.C. \& Rank, R.C. (1984) Effects of counselor and peer-led group assertive training on black-adolescent aggression. Journal of Counseling Psychology 31, 95-98. 
Jaycox, L. et al. (1994) Prevention of depressive symptoms in school children. Behavioral Research and Therapy 32, 801-816.

Jensen, P.S. et al. (2001) Findings from the NIMH multimodal treatment study of ADHD (MTA): Implications and applications for primary care providers. Journal of Developmental and Behavioral Pediatrics 22, 60-73.

Jordans, M.J.D. et al. (2010) Evaluation of a classroom-based psychosocial intervention in conflict-afflicted Nepal: A cluster randomized controlled trial. Journal of Child Psychology and Psychiatry 51, 818-826.

Kahn, J. et al. (1990) Comparison of cognitive-behavioral, relaxation, and self-modeling interventions for depression among middle-school students. School Psychology Review 19, $196-211$.

Kataoka, S.H. et al. (2003) A school-based mental health program for traumatized Latino immigrant children. Journal of the American Academy of Child and Adolescent Psychiatry 42, 311-318.

Kaufman, N.K. et al. (2005) Potential mediators of cognitive-behavioral therapy for adolescents with co-morbid major depression and conduct disorder. Journal of Consulting and Clinical Psychology 73, 38-46.

Kazdin, A.E. (2000) Psychotherapy for Children and Adolescents: Directions for Research and Practice Oxford University Press, New York, NY.

Kazdin, A.E. (2007) Mediators and mechanisms of change in psychotherapy research. Annual Review of Clinical Psychology 3, 1-27.

Kazdin, A.E. (2010) Problem-solving skills training and parent management training for oppositional defiant disorder and conduct disorder. In: Evidence-Based Psychotherapies for 
Children and Adolescents. (eds J.R. Weisz \& A.E. Kazdin), 2nd edn, pp. 211-226. Guilford Press, New York, NY.

Kazdin, A.E. (2011) Single-Case Research Designs: Methods for Clinical and Applied Settings Oxford University Press, Oxford, United Kingdom.

Kazdin, A.E., \& Nock, M.K. (2003) Delineating mechanisms of change in child and adolescent therapy: Methodological issues and research recommendations. Journal of Child Psychology and Psychiatry 44, 1116-1129.

Kazdin, A.E. \& Whitley, M.K. (2003) Treatment of parental stress to enhance therapeutic change among children referred for aggressive and antisocial behavior. Journal of Consulting \& Clinical Psychology 71, 504-515.

Kazdin, A.E. \& Blase, S.L. (2011) Rebooting psychotherapy research and practice to reduce the burden of mental illness. Perspectives on Psychological Science 6, 21-37.

Kazdin, A.E. et al. (1990) Empirical and clinical focus of child and adolescent psychotherapy research. Journal of Consulting \& Clinical Psychology 58, 729-740.

Keel, P.K. \& Haedt, A. (2008) Evidence-based psychosocial treatments for eating problems and eating disorders. Journal of Child and Adolescent Clinical Psychology 37, 39-61.

Kendall, P.C. \& Khanna, M.S. (2008) Camp Cope-A-Lot: The Coping Cat DVD, [DVD], Workbook, Ardmore, PA. Available: http://workbookpublishing.com.

Kendall, P.C. \& Treadwell, K.H. (2007) The role of self-statements as a mediator in treatment for youth with anxiety disorders. Journal of Consulting and Clinical Psychology 75, 380389.

Kendall, P.C. et al. (2011) Computers and psychosocial treatment for child anxiety: Recent advances and ongoing efforts. Depression and Anxiety 28, 58-66. 
Koegel, R.L. et al. (2010) Empirically supported pivotal response treatment for children with autism spectrum disorders. In: Evidence-Based Psychotherapies for Children and Adolescents. (eds J.R. Weisz \& A.E. Kazdin), 2nd edn, pp. 327-344. Guilford Press, New York, NY.

Kraemer, H.C. et al. (2002) Mediators and moderators of treatment effects in randomized clinical trials. Archives of General Psychiatry 59, 877-883.

Lambert, M.J. et al. (2002) Enhancing psychotherapy outcomes via providing feedback on client progress: A replication. Clinical Psychology \& Psychotherapy 9, 91-103.

Lau, W.Y. et al. (2010) Effectiveness of group cognitive-behavioral treatment for childhood anxiety in community clinics. Behaviour Research and Therapy 48, 1067-1077.

Layne, C.M. et al. (2008) Effectiveness of a school-based group psychotherapy program for warexposed adolescents: A randomized controlled trial. Journal of the American Academy of Child and Adolescent Psychiatry 47, 1048-1062.

Le Grange, D. et al. (2012) Moderators and mediators of remission in family-based treatment and adolescent focused therapy for anorexia nervosa. Behaviour Research and Therapy 50, $85-92$.

Leve, L.D. \& Chamberlain, P. (2007) A randomized evaluation of multidimensional treatment foster care: Effects on school attendance and homework completion in juvenile justice girls. Research on Social Work Practice 17, 657-663.

Levitt, E.E. (1957) The results of psychotherapy with children: An evaluation. Journal of Consulting Psychology 21, 189-196.

Levitt, E.E. (1963) Psychotherapy with children: A further evaluation. Behaviour Research and Therapy 60, 326-329. 
Lewis, C.C. et al. (2009) The role of readiness to change in response to treatment of adolescent depression. Journal of Consulting and Clinical Psychology 77, 422-428.

Lipsey, M.W. \& Wilson, D.B. (1998) Effective intervention for serious juvenile offenders: A synthesis of research. In: Serious \& violent juvenile offenders: Risk factors and successful interventions. (eds R. Loeber, \& D.P. Farrington), pp. 313-345. Sage Publications, Inc., Thousand Oaks, CA.

Lochman, J.E. \& Wells, K.C. (2002) Contextual social-cognitive mediators and child outcome: A test of the theoretical model in the coping power program. Development and Psychopathology 14, 971-993.

Lochman, J.E. et al. (2010) Anger control training for aggressive youth. In: Evidence-Based Psychotherapies for Children and Adolescents. (eds J.R. Weisz \& A.E. Kazdin), 2nd edn, pp. 227-242. Guilford Press, New York, NY.

Lock, J. et al. (2001) A Treatment Manual for Anorexia Nervosa: A Family-Based Approach Guilford Press, New York, NY.

Lock, J. et al. (2005) A comparison of short- and long-term family therapy for adolescent anorexia nervosa. Journal of the American Academy of Child and Adolescent Psychiatry 44, $632-639$.

Lonigan, C.J. et al. (1998) Empirically supported psychosocial interventions for children: An overview. Journal of Clinical Child Psychology 27, 138-145.

Lundahl, B. et al. (2006) A meta-analysis of parent training: Moderators and follow-up effects. Clinical Psychology Review 26, 86-104.

Lustig, S.L. et al. (2004) Review of child and adolescent refugee mental health. Journal of the American Academy of Child \& Adolescent Psychiatry 43, 24-36. 
Mather, M. \& Pollard, K. (2009) U.S. Hispanic and Asian population growth levels off, [Online], Available: www.prb.org/Articles/2009/hispanicasian.aspx?p=1 [May 11, 2009].

Mathur, S.R. et al. (1998) Social skills interventions with students with emotional and behavioral problems: A quantitative synthesis of single-subject research. Behavioral Disorders 23, 193201.

McGrath, M.L. et al. (1987) "Beat-the-buzzer": A method for decreasing parent-child morning conflicts. Child and Family Behavior Therapy 9(3-4), 35-48.

McLeod, B.D. (2011) Relation of the alliance with outcomes in youth psychotherapy: A metaanalysis. Clinical Psychology Review 31, 603-616.

McLeod, B.D. \& Weisz, J.R. (2005) The Therapy Process Observational Coding System—Alliance scale: Measure characteristics and prediction of outcome in usual clinical practice. Journal of Consulting and Clinical Psychology 73, 323-333.

McLeod, B.D. \& Weisz, J.R. (2010) Therapy process observational coding system for child psychotherapy strategies scale. Journal of Clinical Child and Adolescent Psychology 39, 436-443.

McMahon, R.J. \& Forehand, R. (2003) Helping the Noncompliant Child: Family Based Treatment for Oppositional Behavior Guilford Press, New York, NY.

Merry, S.N. et al. (2012) The effectiveness of SPARX, a computerized self help intervention for adolescents seeking help for depression: randomized controlled non-inferiority trial. British Medical Journal 344, 1-16.

Mihalic, S. et al. (2002) Blueprints for Violence Prevention Replications: Factors for Implementation Success Center for the Study and Prevention of Violence, Boulder, CO. 
MTA Cooperative Group (1999) A 14-month randomized clinical trial of treatment strategies for attention-deficit/hyperactivity disorder. Archives of General Psychiatry 56, 1073-1086.

Mufson, L.H. et al. (1999) Efficacy of interpersonal therapy for depressed adolescents. Archives of General Psychiatry 56, 573-579.

NIMH (2009) Dissemination and implementation research in health (R21), [Online], Available: http://grants.nih.gov/grants/guide/pa-files/par-10-040.html [14 Mar 2013].

Palinkas, L.A. et al. (2008) An ethnographic study of implementation of evidence-based practice in child mental health: First steps. Psychiatric Services 59, 738-746.

Patel, V. et al. (2008) Promoting child and adolescent mental health in low and middle income countries. Journal of Child Psychology and Psychiatry 49, 313-334.

Pelham, W.E. \& Fabiano, G.A. (2008) Evidence-based psychosocial treatments for attentiondeficit/hyperactivity disorder. Journal of Clinical Child and Adolescent Psychology 37, 184214.

Pelham, W.E. et al. (2010) Summer treatment programs for attention-deficit/hyperactivity disorder. In: Evidence-Based Psychotherapies for Children and Adolescents. (eds J.R. Weisz \& A.E. Kazdin), 2nd edn, pp. 277-292. Guilford Press, New York, NY.

Rogers, S.J. \& Vismara, L.A. (2008) Evidence-based comprehensive treatments for early autism. Journal of Child and Adolescent Clinical Psychology 37, 8-38.

Sanders, M.R. \& Murphy-Brennan, M. (2010) The international dissemination of the triple PPositive parenting program. In: Evidence-Based Psychotherapies for Children and Adolescents. (eds J. R. Weisz \& A. E. Kazdin), 2nd edn, pp. 519-537. Guilford Press, New York, NY. 
Saxena, S. et al. (2006) The 10/90 divide in mental health research: Trends over a 10-year period. The British Journal of Psychiatry 188, 81-82.

Schoenwald, S.K. (2010) From policy pinball to purposeful partnership: The policy contexts of Multisystemic Therapy transport and dissemination. In: Evidence-based psychotherapies for children and adolescents. (eds J. R. Weisz \& A. E. Kazdin), 2nd edn, pp. 538-553. Guilford Press, New York, NY.

Scott, S. (2013, May 31). Section leader presentation in: Translation of evidence-based knowledge into real-world settings. Marbach Castle Conference, Lake Constance, Germany.

Scott, S., \& Dadds, M.R. (2009) Practitioner review: When parent training doesn't work: theorydriven clinical strategies. Journal of Child Psychology and Psychiatry 50, 1441-1450.

Scott, S. (Chair) \& Guideline Development Group Members (2013) Antisocial Behavior and Conduct Disorders in Children and Young People: Recognition, Intervention and Management National Institute for Health and Clinical Excellence, London, United Kingdom.

Shapiro, D.A. \& Shapiro, D. (1982) Meta-analysis of comparative therapy outcome studies: A replication and refinement. Psychological Bulletin 92, 581-604.

Shaw, J.A. (2003) Children exposed to war/terrorism. Clinical Child and Family Psychology Review 6, 237-246.

Silverman, W.K. \& Hinshaw, S.P. (2008) The second special issue on evidence-based psychosocial treatments for children and adolescents: A 10-year update. Journal of Child and Adolescent Clinical Psychology 37, 1-7. 
Silverman, W.K. et al. (2008a) Evidence-based psychosocial treatments for children and adolescents exposed to traumatic events. Journal of Child and Adolescent Clinical Psychology 37, 156-183.

Silverman, W.K. et al. (2008b) Evidence-based psychosocial treatments for phobic and anxiety disorders in children and adolescents. Journal of Child and Adolescent Clinical Psychology $37,105-130$.

Smith, D.K. \& Chamberlain, P. (2010) Multidimensional treatment foster care for adolescents: Processes and outcomes. In: Evidence-Based Psychotherapies for Children and Adolescents. (eds J.R. Weisz \& A.E. Kazdin), 2nd edn, pp. 243-258. Guilford Press, New York, NY.

Smith, M.L. \& Glass, G.V. (1977) Meta-analysis of psychotherapy outcome studies. American Psychologist 32, 752-760.

Smith, T. (2010) Early and intensive behavioral intervention in autism. In: Evidence-Based Psychotherapies for Children and Adolescents. (eds J.R. Weisz \& A.E. Kazdin), 2nd edn, pp. 312-326. Guilford Press, New York, NY.

Sonuga-Barke, E.J.S. et al. (2013) Nonpharmacological interventions for ADHD: Systematic review and meta-analysis of randomized controlled trials of dietary and psychological treatment. American Journal of Psychiatry 170, 275-289.

Southam-Gerow, M.A. et al. (2003) Youth with anxiety disorders in research and service clinics: Examining client differences and similarities. Journal of Clinical Child and Adolescent Psychology 32, 375-385.

Southam-Gerow, M.A. et al. (2010) Does CBT for youth anxiety outperform usual care in community clinics? An initial effectiveness test. Journal of the American Academy of Child \& Adolescent Psychiatry 49, 1043-1052. 
Spielmans, G.I. et al. (2010) The efficacy of evidence-based psychotherapies versus usual care for youths: Controlling confounds in a meta-reanalysis. Psychotherapy Research 20, 234246.

Stagman, S. \& Cooper, J.L. (2010) Children's Mental Health: What Every Policymaker Should Know National Center for Children in Poverty, Columbia University Mailman School of Public Health, New York, NY.

Stein, B.D. et al. (2003) A mental health intervention for schoolchildren exposed to violence. Journal of the American Medical Association 290, 603-611.

Stein, B.D. et al. (2010) Use of outcomes information in child mental health treatment: Results from a pilot study. Psychiatric Services 61, 1211-1216.

Stice, E. et al. (2010) Testing mediators of intervention effects in randomized controlled trials: An evaluation of three depression prevention programs. Journal of Consulting and Clinical Psychology 78, 273-280.

Swenson, C.C. et al. (2010) Multisystemic Therapy for Child Abuse and Neglect: A randomized effectiveness trial. Journal of Family Psychology 24, 497-507.

Tarnowski, K.J. et al. (1987) A modified habit reversal procedure in a recalcitrant case of trichotillomania. Journal of Behavior Therapy and Experimental Psychiatry 18, 157-163.

Teplin, L.A. et al. (2006) Psychiatric disorders of youth in detention U.S. Department of Justice, Washington, DC.

Tol, W.A. et al. (2008) School-based mental health intervention for children affected by political violence in Indonesia: A cluster randomized trial. Journal of the American Medical Association 300, 655-662. 
Tolan, P.H. et al. (2009) The benefits of booster interventions: Evidence from a family-focused prevention program. Prevention Science 10, 287-297.

Treadwell, K.H. \& Kendall, P.C. (1996) Self-talk in youth with anxiety disorders: States of mind, content specificity. Journal of Consulting and Clinical Psychology 64, 941-950.

Treatment for Adolescents With Depression Study (TADS) Team (2004) Fluoxetine, cognitive behavioral therapy, and their combination for adolescents with depression: Treatment for adolescents with depression study (TADS) randomized controlled trial. Journal of the American Medical Association 292, 807-820.

U.S. Department of Health and Human Services (2001) Mental health: Culture, race, and ethnicity-A supplement to Mental Health: A Report of the Surgeon General U.S. Department of Health and Human Services, Public Health Service, Office of the Surgeon General, Rockville, MD.

Waldron, H.B. \& Kaminer, Y. (2004) On the learning curve: The emerging evidence supporting cognitive-behavioral therapies for adolescent substance abuse. Addiction 99(Suppl. 2), 93105.

Waldron, H.B. \& Turner, C.W. (2008) Evidence-based psychosocial treatments for adolescent substance abuse. Journal of Child and Adolescent Clinical Psychology 37, 238-261.

Watanabe, N. et al. (2007) Psychotherapy for depression among children and adolescents: A systematic review. Acta Psychiatrica Scandinavica 116, 84-95.

Webb, C. et al. (2010) Therapist adherence/competence and treatment outcome: A meta-analytic review. Journal of Consulting and Clinical Psychology 78, 200-211.

Webster-Stratton, C. \& Reid, M.J. (2010) The incredible years parents, teachers, and children's training series: A multifaceted treatment approach for young children with conduct problems. 
In: Evidence-Based Psychotherapies for Children and Adolescents. (eds J.R. Weisz \& A.E. Kazdin), 2nd edn, pp. 194-210. Guilford Press, New York, NY.

Weersing, V.R. \& Weisz, J.R. (2002a) Community clinic treatment of depressed youth:

Benchmarking usual care against CBT clinical trials. Journal of Consulting and Clinical Psychology 70, 299-310.

Weersing, V.R. \& Weisz, J.R. (2002b) Mechanisms of action in youth psychotherapy. Journal of Child Psychology and Psychiatry 43, 3-29.

Weersing, V.R. et al. (2002) Development of the therapy procedures checklist: A therapist-report measure of technique use in child and adolescent treatment. Journal of Clinical Child and Adolescent Psychology 31, 168-180.

Weisz, J.R. (2004) Psychotherapy for Children and Adolescents: Evidence-Based Treatments and Case Examples Cambridge University Press, Cambridge, United Kingdom.

Weisz, J.R. \& Addis, M.E. (2006) The research-practice tango and other choreographic challenges: Using and testing evidence-based psychotherapies in clinical care settings. In: Evidence-Based Psychotherapy: Where Practice and Research Meet. (eds C.D. Goodheart, A.E. Kazdin, \& R.J. Sternberg), pp. 179-206, American Psychological Association, Washington, DC.

Weisz, J.R. \& Gray, J.S. (2008) Evidence-based psychotherapies for children and adolescents: Data from the present and a model for the future. Child and Adolescent Mental Health 13, $54-65$.

Weisz, J.R. \& Kazdin, A.E. (eds) (2010) Evidence-Based Psychotherapies for Children and Adolescents, 2nd edn. Guilford Press, New York, NY. 
Weisz, J.R. et al. (1987) Effectiveness of psychotherapy with children and adolescents: A metaanalysis for clinicians. Journal of Consulting \& Clinical Psychology 55, 542-549.

Weisz, J.R. et al. (1995) Effects of psychotherapy with children and adolescents revisited: A meta-analysis of treatment outcome studies. Psychological Bulletin 117, 450-468.

Weisz, J.R. et al. (2005a) Youth psychotherapy outcome research: A review and critique of the evidence base. Annual Review of Psychology 56, 337-363.

Weisz, J.R. et al. (2005b) Promoting and protecting youth mental health through evidence-based prevention and treatment. American Psychologist 60, 628-648.

Weisz, J.R. et al. (2006a) Evidence-based youth psychotherapies versus usual clinical care: A meta-analysis of direct comparisons. American Psychologist 61, 671-689.

Weisz, J.R. et al. (2006b) Effects of psychotherapy for depression in children and adolescents: A meta-analysis. Psychological Bulletin 132, 132-149.

Weisz, J.R. et al. (2009) Cognitive-behavioral therapy versus usual clinical care for youth depression: An initial test of transportability to community clinics and clinicians. Journal of Consulting and Clinical Psychology 77, 383-396.

Weisz, J.R. et al. (2012) Testing standard and modular designs for psychotherapy with youth depression, anxiety, and conduct problems: A randomized effectiveness trial. Archives of General Psychiatry 69, 274-282.

Weisz, J.R. et al. (2013a) Evidence-based youth psychotherapy in the mental health ecosystem. Journal of Clinical Child \& Adolescent Psychology 42, 274-286.

Weisz, J.R. et al. (2013b) Performance of evidence-based youth psychotherapies compared with usual clinical care: A multilevel meta-analysis. JAMA Psychiatry 70, 750-761. 
Wurtele, S.K. \& Drabman, R.S. (1984) "Beat the buzzer” for classroom dawdling: A one-year trial. Behavior Therapy 15, 403-409.

Yu, D.L. \& Seligman, M.E.P. (2002) Preventing depressive symptoms in Chinese children. Prevention and Treatment, 5, Article 9.

Zisser, A. \& Eyberg, S.M. (2010) Parent-child interaction therapy and the treatment of disruptive behavior disorders. In: Evidence-Based Psychotherapies for Children and Adolescents. (eds J.R. Weisz \& A.E. Kazdin), 2nd edn, pp. 179-193. Guilford Press, New York, NY. 


\section{Table 1}

\section{Evidence-based Youth Psychotherapies ${ }^{1,2}$}

\begin{tabular}{|c|c|}
\hline Problem/Disorder Category & Well-established (WE) or Probably Efficacious (PE) Psychotherapy \\
\hline $\begin{array}{l}\text { Early Autism (Rogers \& Vismara } \\
\text { 2008) }\end{array}$ & $\begin{array}{l}\text { - } \quad \text { Lovaas Model: Intensive Behavioral Intervention (WE) } \\
\text { - } \quad \text { Pivotal Response Treatment (PE) }\end{array}$ \\
\hline $\begin{array}{l}\text { Eating Disorders in Adolescence } \\
\text { (Keel \& Haedt 2008) }\end{array}$ & - $\quad$ Family Therapy for Anorexia Nervosa (WE) \\
\hline $\begin{array}{l}\text { Depression (David-Ferdon \& } \\
\text { Kaslow 2008) }\end{array}$ & $\begin{array}{l}\text { - } \text { CBT for Children (WE) } \\
\text { - } \text { CBT for Adolescents (WE) } \\
\text { - } \quad \text { Interpersonal Psychotherapy for Adolescents (WE) } \\
\text { - Behavior Therapy for Children (PE) }\end{array}$ \\
\hline $\begin{array}{l}\text { Phobic \& Anxiety Disorders } \\
\text { (Silverman et al. 2008b) }\end{array}$ & $\begin{array}{ll}\text { - } & \text { Group CBT (PE) } \\
\text { - } & \text { Group CBT for Social Phobia (PE) } \\
\text { - } & \text { Group CBT with Parents (PE) } \\
\text { - } & \text { Individual CBT (PE) } \\
\text { - } & \text { Social Effectiveness Training for Social Phobia (PE) }\end{array}$ \\
\hline $\begin{array}{l}\text { Obsessive-Compulsive Disorder } \\
\text { (Barrett } \text { et al. 2008) }\end{array}$ & - $\quad$ Individual Exposure-Based CBT (PE) \\
\hline $\begin{array}{l}\text { Youths Exposed to Traumatic } \\
\text { Events (Silverman et al. 2008a) }\end{array}$ & $\begin{array}{ll}\text { - } & \text { Trauma-Focused CBT (WE) } \\
\text { - } & \text { School-Based Group CBT (PE) }\end{array}$ \\
\hline $\begin{array}{l}\text { Attention-Deficit/Hyperactivity } \\
\text { Disorder (Pelham \& Fabiano } \\
\text { 2008) }\end{array}$ & $\begin{array}{ll}- & \text { Behavioral Classroom Management (WE) } \\
\text { - } & \text { Behavioral Parent Training (WE) } \\
\text { - } & \text { Intensive Peer-Focused Behavioral Interventions in Recreational } \\
& \text { Settings (WE) }\end{array}$ \\
\hline $\begin{array}{l}\text { Disruptive Behavior (Eyberg et } \\
\text { al. 2008) }\end{array}$ & $\begin{array}{l}\text { - } \text { Parent Management Training Oregon Model (WE) } \\
\text { - } \quad \text { Anger Control Training (PE) }\end{array}$ \\
\hline
\end{tabular}




\begin{tabular}{|c|c|}
\hline & $\begin{array}{l}\text { - } \text { Group Assertive Training (PE) } \\
\text { - } \quad \text { Helping the Noncompliant Child (PE) } \\
\text { - } \quad \text { Incredible Years Parent Training (PE) } \\
\text { - } \quad \text { Incredible Years Child Training (PE) } \\
\text { - } \quad \text { Multidimensional Treatment Foster Care (PE) } \\
\text { - } \quad \text { Multisystemic Therapy (PE) } \\
\text { - } \quad \text { Parent-Child Interaction Therapy (PE) } \\
\text { - } \quad \text { Positive Parenting Program-Standard (PE) } \\
\text { - } \quad \text { Positive Parenting Program-Enhanced (PE) } \\
\text { - } \quad \text { Problem-Solving Skills Training (PSST) (PE) } \\
\text { - } \quad \text { PSST + Practice (PE) } \\
\text { - } \quad \text { PSST + Parent Management Training (PE) } \\
\text { - } \quad \text { Rational-Emotive Mental Health Program (PE) }\end{array}$ \\
\hline $\begin{array}{l}\text { Adolescent Substance Abuse } \\
\text { (Waldron \& Turner 2008)) }\end{array}$ & $\begin{array}{l}\text { - } \text { Functional Family Therapy } \\
\text { - } \quad \text { Group CBT (WE) } \\
\text { - } \quad \text { Individual CBT (WE) } \\
\text { - } \quad \text { Multidimensional Family Therapy (WE) } \\
\text { - } \quad \text { Behavioral Family Therapy (PE) } \\
\text { - } \quad \text { Brief Strategic Family Therapy (PE) } \\
\text { - } \quad \text { Multisystemic Therapy (PE) }\end{array}$ \\
\hline
\end{tabular}

${ }^{1}$ From "Evidence-based psychosocial treatments for children and adolescents: A ten-year update.” Journal of Clinical Child and Adolescent Psychology, 37, 1-262.

${ }^{2}$ This table shows classifications for broad forms of psychotherapy (e.g., Cognitive-Behavioral Therapy $[\mathrm{CBT}])$; some reports in the special issue of the journal also classified specific treatment subtypes (e.g., group CBT for children, individual adolescent CBT plus parent/family component), which are not included in the table, given space limitations. 
Table 2

Percentage of Groups in Youth Psychotherapy Outcome Studies that Employed Clinically Representative Youths, Therapists, and Treatment Settings

\begin{tabular}{|c|c|c|c|c|c|c|}
\hline Decade & $1960 s$ & $1970 \mathrm{~s}$ & 1980s & $1990 \mathrm{~s}$ & $2000 \mathrm{~s}$ & $\begin{array}{c}\text { All } \\
\text { Decades }\end{array}$ \\
\hline No. of Studies & 13 & 62 & 99 & 100 & 187 & 461 \\
\hline No. of Groups & 35 & 183 & 273 & 244 & 425 & 1160 \\
\hline \multicolumn{7}{|l|}{ How Youths Were Enrolled in Study } \\
\hline Recruited, non-treatment-seeking & 62.9 & 85.8 & 65.9 & 57.8 & 62.8 & 66.1 \\
\hline Clinic-referred, treatment-seeking & 5.7 & 4.9 & 24.2 & 26.2 & 24.5 & 21.1 \\
\hline Required via court/justice system & 17.1 & 8.2 & 8.8 & 11.5 & 7.8 & 9.1 \\
\hline Enrollment method not reported & 14.3 & 1.1 & 1.1 & 4.5 & 4.9 & 3.6 \\
\hline
\end{tabular}

Who Provided the Treatment

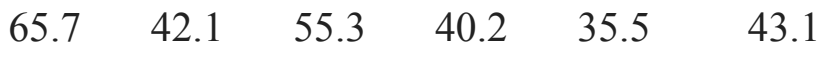

$>50 \%$ therapists are practitioners

$\begin{array}{llllll}2.9 & 9.8 & 7.7 & 10.7 & 19.1 & 12.7\end{array}$

Therapist vocation not reported

$\begin{array}{llllll}14.3 & 27.9 & 23.4 & 32.0 & 25.4 & 26.4\end{array}$

No treatment/waitlist

$\begin{array}{llllll}17.1 & 20.2 & 13.6 & 17.2 & 20.0 & 17.8\end{array}$

Where Treatment Took Place
Research setting
$11.4 \quad 6.6$
9.9
$18.4 \quad 19.5$
14.7
Custodial, school, supervised setting

$\begin{array}{llllll}8.6 & 29.5 & 32.6 & 20.5 & 22.6 & 25.2\end{array}$




$\begin{array}{lcccccc}\text { Clinical service setting } & 0 & 2.7 & 5.9 & 11.5 & 14.8 & 9.7 \\ \text { Correctional setting } & 14.3 & 1.1 & 5.1 & 4.1 & 2.1 & 3.4 \\ \text { Treatment setting not reported } & 48.6 & 39.9 & 33.0 & 28.3 & 20.9 & 29.1 \\ \text { No treatment/waitlist } & 17.1 & 20.2 & 13.6 & 17.2 & 20.0 & 17.8\end{array}$

Number of Representativeness Factors

No factors reported

One factor reported

Two factors reported

Three factors reported
91.4

8.

0

0

\section{$8.6 \quad 15.3$}

$0 \quad 1$.

$1.1 \quad 7.0$

0

0.4

70.0

63.9

61.2

68.3

$22.7 \quad 25.4 \quad 23.8$

22.1

$\begin{array}{lll}9.0 & 10.6 & 7.6\end{array}$

Note. Because treatment provider and setting are group-level variables, percentages of groups rather than percentages of studies are reported for all three variables. Three studies that employed a combination of non-treatment seeking and treatment seeking youths, and one study with a treatment condition that employed a combination of clinical and research settings, were excluded from analyses. [Reprinted with permission, John R. Weisz] 


\section{Figure Captions}

Figure 1. Mean effect sizes found in two broad-based meta-analyses of adult psychotherapy effects (the two bars at the left: Smith \& Glass, 1977; Shapiro \& Shapiro, 1982), four broadbased meta-analyses of youth psychotherapy effects (the four middle bars: Casey \& Berman, 1985; Weisz et al., 1987; Kazdin et al., 1990; Weisz et al., 1995), and two meta-analyses of RCTs comparing evidence-based youth psychotherapies to usual clinical care (the two bars at the right: Weisz et al. 2006a and 2013b). The full bar for Kazdin et al. (1990) shows the mean effect size for treatment vs. inert control group comparisons; the dashed line shows the mean for treatment vs. active control group comparisons. The full bar for Weisz et al. (1995) shows the mean effect size when unweighted least squares analyses were conducted; the dashed line shows the mean for weighted least squares analyses. [Reprinted with permission, John R. Weisz]

Figure 2. Effect sizes of individual studies comparing evidence-based youth psychotherapies (EBPs) to usual care. Horizontal bar at .29 shows mean effect size across the full study set. Bars below zero show studies in which usual care produced outcomes superior to those of EBPs. Note the number of studies for which usual care showed effects similar to or superior to EBPs. [Reprinted with permission, John R. Weisz] 


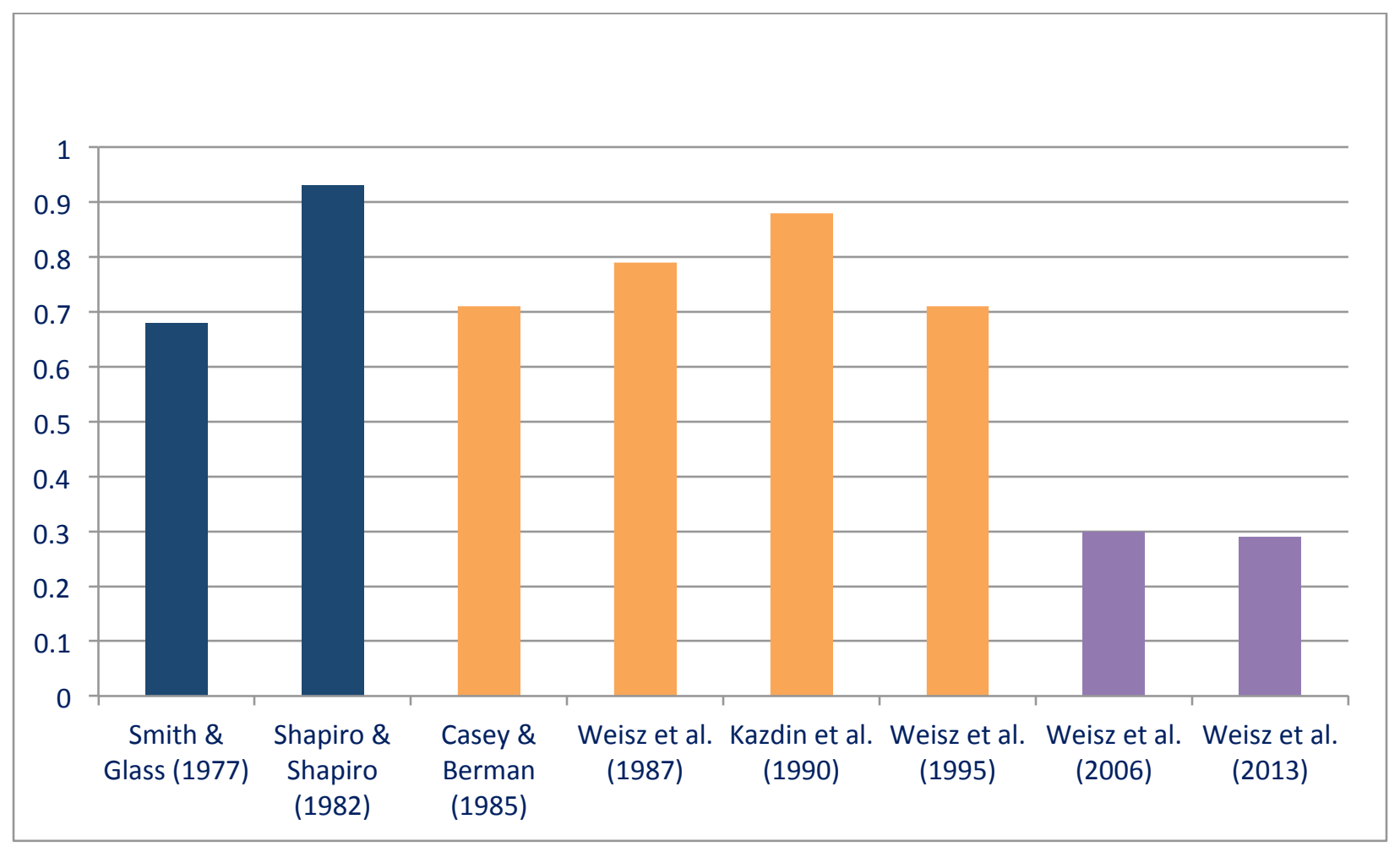




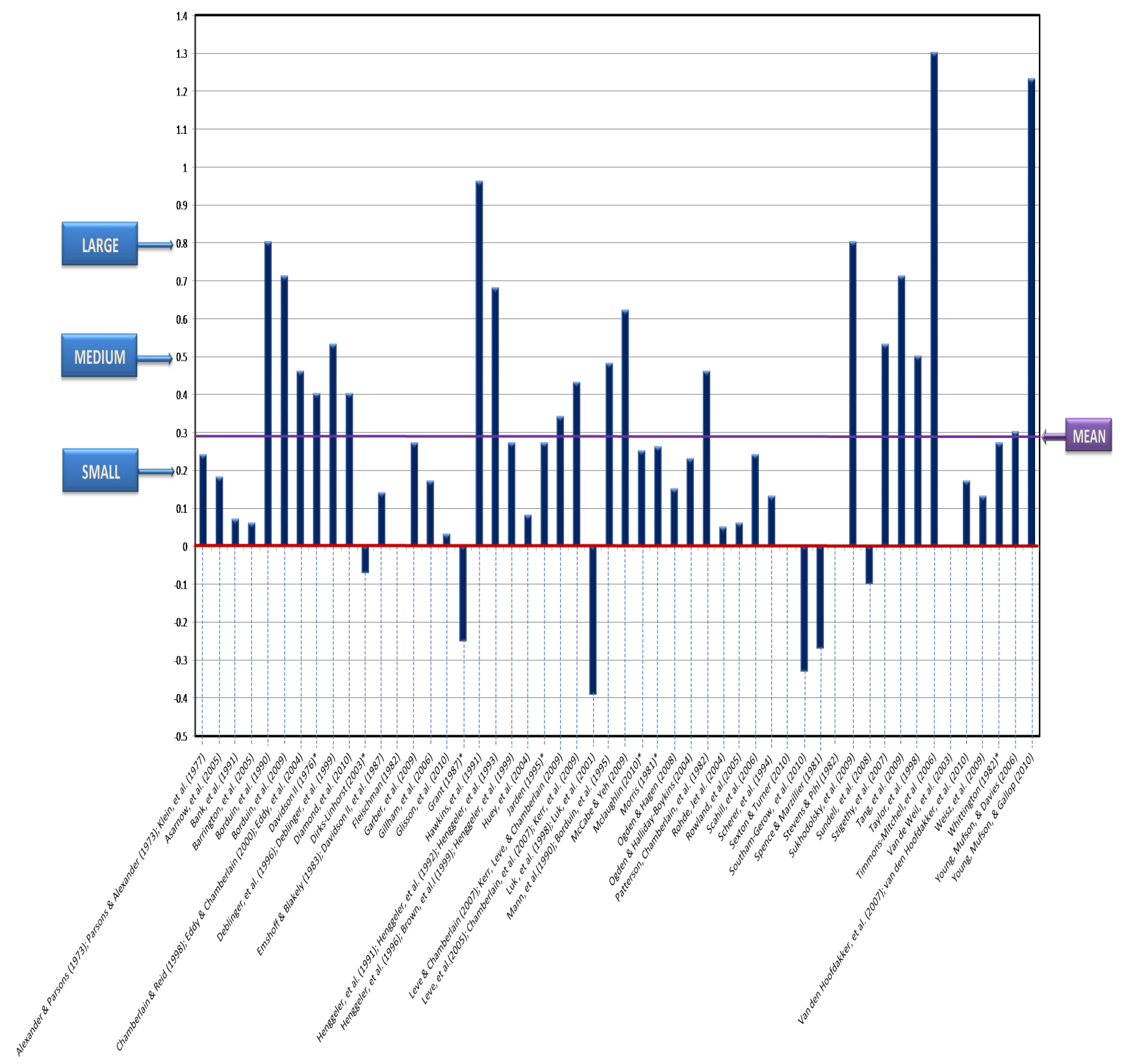

